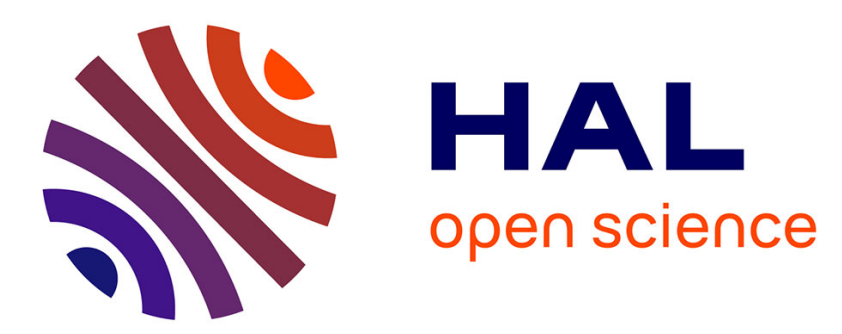

\title{
A geometric dissimilarity criterion between Jordan spatial mosaics. Theoretical aspects and application to segmentation evaluation \\ Yann Gavet, Jean-Charles Pinoli
}

\section{- To cite this version:}

Yann Gavet, Jean-Charles Pinoli. A geometric dissimilarity criterion between Jordan spatial mosaics. Theoretical aspects and application to segmentation evaluation. Journal of Mathematical Imaging and Vision, 2012, 42 (1), pp.25-49. 10.1007/s10851-011-0272-4 . hal-00660178

\section{HAL Id: hal-00660178 https://hal.science/hal-00660178}

Submitted on 16 Jan 2012

HAL is a multi-disciplinary open access archive for the deposit and dissemination of scientific research documents, whether they are published or not. The documents may come from teaching and research institutions in France or abroad, or from public or private research centers.
L'archive ouverte pluridisciplinaire HAL, est destinée au dépôt et à la diffusion de documents scientifiques de niveau recherche, publiés ou non, émanant des établissements d'enseignement et de recherche français ou étrangers, des laboratoires publics ou privés. 


\title{
A geometric dissimilarity criterion between Jordan spatial mosaics. Theoretical aspects and application to segmentation evaluation.
}

\author{
Yann Gavet · Jean-Charles Pinoli
}

the date of receipt and acceptance should be inserted later

\begin{abstract}
An image segmentation process often results in a special spatial set, called a mosaic, as the subdivision of a domain $S$ within the $n$-dimensional Euclidean space. In this paper, $S$ will be a compact domain and the study will be focused on finite Jordan mosaics, that is to say mosaics with a finite number of regions and where the boundary of each region is a Jordan hypersurface.

The first part of this paper addresses the problem of comparing a Jordan mosaic to a given reference Jordan mosaic and introduces the $\epsilon$ dissimilarity criterion. The second part will show that the $\epsilon$ dissimilarity criterion can be used to perform the evaluation of image segmentation processes. It will be compared to classical criterions in regard to several geometric transformations. The pros and cons of these criterions are presented and discussed, showing that the $\epsilon$ dissimilarity criterion outperforms the other ones.
\end{abstract}

Keywords Dissimilarity criterion · Geometric Distances · Spatial mosaics · Human visual perception · Supervised segmentation evaluation.

CIS and LPMG, UMR CNRS 5148, IFRESIS, IFR INSERM 143,

École Nationale Supérieure des Mines de Saint-Etienne, 158 Cours Fauriel, 42023 Saint-Etienne cedex, France Tel.: +3347742 0170; fax: +33477499694.

E-mail address: E-mail: gavet@emse.fr 


\section{Part I: Theoretical aspects}

\section{Introduction}

\subsection{Spatial mosaics in $\mathbb{R}^{n}$}

Let $S$ be a non-empty closed bounded subset of the $n$-dimensional Euclidean space, denoted $\mathbb{R}^{n}$. In this article, $S$ is simply connected and its interior is non empty. Its boundary will be supposed to be a Jordan hypersurface, or simply called a surface $([8,21])$. Mathematically, this means that $S$ is homeomorphic to the unit ball in $\mathbb{R}^{n}$. The set $S$ designates the working domain in which Jordan mosaics will be studied. It will be thus called the domain of interest. Practically, $S$ will be for example a rectangle in the two-dimensional Euclidean space $\mathbb{R}^{2}$ (Fig. 1).

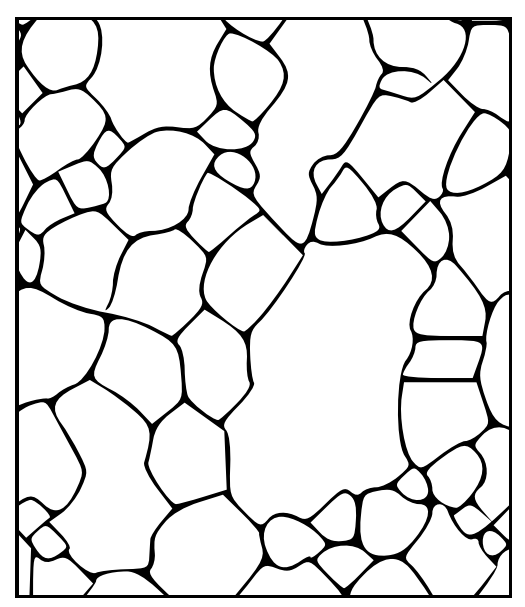

Fig. 1 This figure is a view that shows a piece of a mosaic.

A mosaic can then be defined on $S$ as follows: it is constituted of disjoined adjacent connected open subsets called regions, denoted $R_{i}$ for $i \in I$ ( $I$ is the index set), so that the closure of their union set equals the whole domain $S$. The collection of such regions for a given domain $S$ will be supposed to be finite and indexed by a natural number range $I \subset \mathbb{N}$. A region $R_{i}$ (for $i \in I$ ) is in fact defined by its boundary $\partial R_{i}$, which is supposed to be a Jordan (hyper)surface (a Jordan curve in the two-dimensional case). A Jordan surface is a simple closed continuous surface that separates the space $\mathbb{R}^{n}$ into two separated open connected subsets ([21]). Practically, this surface has mathematical nice properties that correspond to what is visually seen in $\mathbb{R}^{2}$ (see Fig. 2).

Definition 1 (Jordan mosaic).

A Jordan mosaic $M$ is the set of contours $C=\bigcup \partial R_{i}, i \in I$, defined as follows:

$$
\forall(i, j) \in I \text { (index set) }, i \neq j, R_{i} \cap R_{j}=\emptyset
$$

and

$$
\left.\begin{array}{l}
R=\bigcup\left\{R_{i}\right\}, i \in I \\
C=\bigcup\left\{\partial R_{i}\right\}, i \in I \\
S=R \bigcup C \\
\emptyset=R \cap C
\end{array}\right\}
$$

where each boundary $\partial R_{i}$ is a Jordan surface.

Notice that there is a duality between regions $\left(R=\bigcup\left\{R_{i}\right\}\right)$ and contours $\left(C=\cup \partial R_{i}\right)$ (Eq. 2). 
Tesselations are special mosaics where the regions are polyhedra. The classical references $[14,45,46,47,38]$ considered regular tesselations of planes and higher-dimensional spaces. See [32] for a historical sketch of the ideas development .

Mosaics addressed in the present paper are more general since each region is neither restricted to a polyhedron nor a simply connected set (Fig. 2).

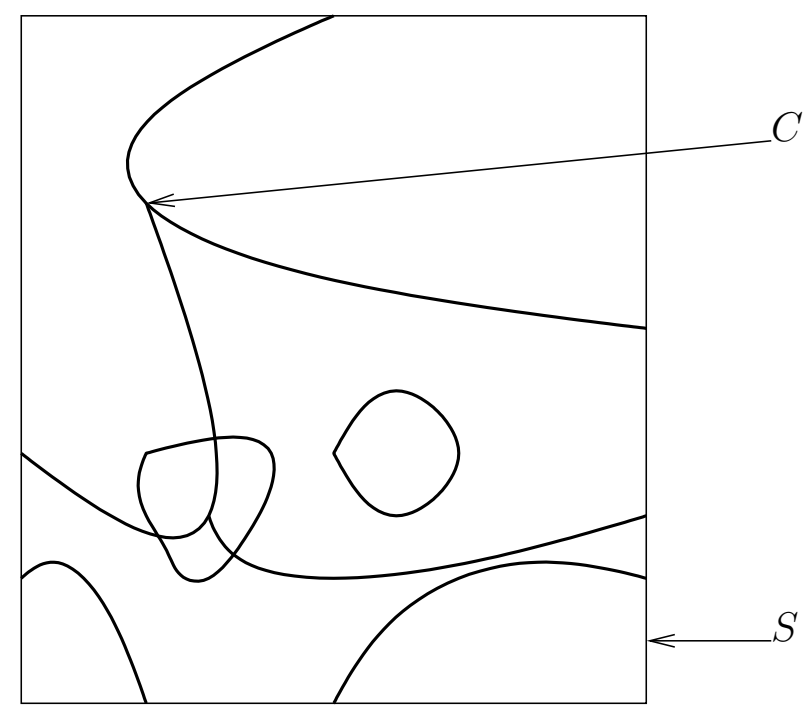

Fig. 2 Spatial Jordan mosaic example in $\mathbb{R}^{2}$. The domain of interest $S$ (a rectangle) is separated into several disjoined adjacent and connected regions $\left(R_{i}\right)_{i \in I}$ by their contours $C$.

\subsection{Aim and outline of this first part}

The comparison methods for spatial mosaics fall into two distinct categories: region-based or contour-based approaches. The region-based approaches consider a collection of regions as described above. In this case, a comparison of two mosaics basically consists on making a one-to-one correspondance between regions of each mosaic, which is generally not possible.

This first part aims at comparing spatial Jordan mosaics together from the contours "point of view", and more precisely to compare such a mosaic to a given reference spatial Jordan mosaic. First, some classical distance functions used to evaluate discrepancies between Euclidean sets will be presented, namely the three classical metrics: Hausdorff, Nikodym and Steinhaus distances, respectively (Sect. 2). Second, it will be shown that these distances are not adapted to mosaic comparison (Sect. 3). Third (Sect. 4), these distances will be extended, but will still remain irrelevant. In Sect. 5, the study of the human visual perception will highlight that the metric notion is too strong, and even not geometrically or visually relevant. The notion of dissimilarity is discussed since it appears to better suit how the visual perception system performs the comparison process. Sect. 6 presents a novel geometric dissimilarity criterion that allows to perform the mosaic comparison. In Sects. 7 and 8, its properties and asymptotic behavior are studied.

\section{Geometric Distances in $\mathbb{R}^{n}$}

This section first recalls the definition of a peculiar distance function called a metric and then presents three metrics classically used to compare (rigid) geometric sets. 


\subsection{Definition}

Distance functions ([13]) are functionals adapted to perform comparisons of (mathematical) objects belonging to the same family $\xi$ (for example, $\xi$ is the family of the Jordan mosaics on a given reference domain $S$ ).

\section{Definition 2 (Metric).}

A metric is a particular distance function $d$ that is a function from $\xi^{2}$ into $\mathbb{R}_{+}$and verifies the four following axioms (see [13]):

$$
\begin{array}{r}
\text { (identity) } \\
\forall x \in \xi, \quad d(x, x)=0 \\
\text { (separation) } \\
\forall x, y \in \xi, \quad d(x, y)=0 \Rightarrow x=y \\
\text { (symmetry) } \\
\forall x, y \in \xi, \quad d(x, y)=d(y, x) \\
\text { (triangle inequality) } \\
\forall x, y, z \in \xi, d(x, y) \leq d(x, z)+d(z, y)
\end{array}
$$

These axioms are mathematically important and will be discussed in Sect. 5 .

\subsection{Three classical geometric distances}

This article does not aim to give an exhaustive overview of distances. The reader will refer to [13] for a deep review. One classical metric used between compact sets in $\mathbb{R}^{n}$ is the Hausdorff distance ([34,18]). Another distance, called the Nikodỳm distance $([31])$, is based on the Lebesgue measure of the symmetric difference between measurable sets. A derived distance called the Steinhaus distance has been historically next defined $([23])$.

Definition 3 (Hausdorff half-distance).

Let $d_{E}$ be the Euclidean metric and $S$ a given domain in $\mathbb{R}^{n}$. If the application $d_{E}(a, B)$ denotes the Euclidean distance between a point $a \in S \subset \mathbb{R}^{n}$ and a compact set $B \subset S$, then the Hausdorff half-distance denoted $f_{d_{E}}$ between two compact sets $A$ and $B(A, B \subset S)$ is defined by Eq. 7:

$$
\begin{aligned}
f_{d_{E}}(A, B) & =\sup _{a \in A} d_{E}(a, B) \\
\text { with } d_{E}(a, B) & =\inf _{b \in B} d_{E}(a, b)
\end{aligned}
$$

\section{Definition 4 (Hausdorff distance).}

The Hausdorff distance (Fig. 3) between two compact sets $A$ and $B$, denoted $d_{H}(A, B)$, is then defined by Eq. 9 (notice that the symmetry property is now satisfied in this equation):

$$
d_{H}(A, B)=\max \left\{f_{d_{E}}(A, B), f_{d_{E}}(B, A)\right\}
$$

The supremum operator in Eq. 7 implies that if only one point is added to $A$ or $B$, the Hausdorff distance value might change a lot.

\section{Definition 5 (Symmetric difference).}

The symmetric difference set between two sets $A$ and $B(A \subset S$ and $B \subset S)$ in $\mathbb{R}^{n}$, denoted $\Delta(A, B)$, is defined by the following equation (Eq. 10) and illustrated in Fig. 4:

$$
\begin{aligned}
\Delta(A, B) & =(A \cup B) \backslash(A \cap B) \\
& =(A \backslash B) \cup(B \backslash A)
\end{aligned}
$$




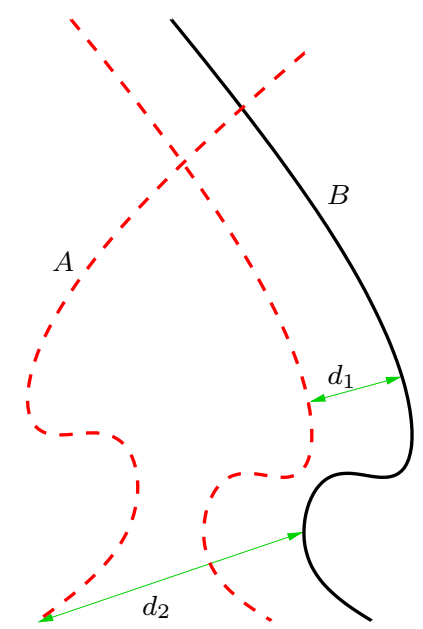

Fig. 3 Illustration of Hausdorff distance between two sets $A$ and $B: d_{1}=f(A, B)$ and $d_{2}=f(B, A)$.

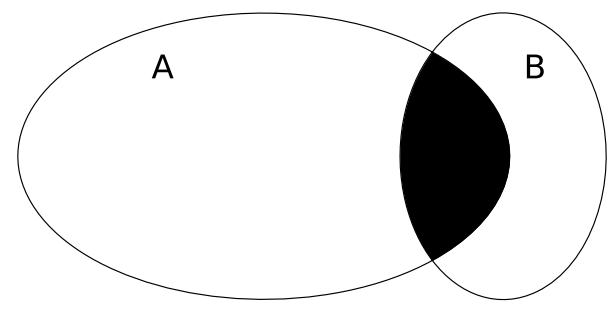

Fig. 4 Illustration of the symmetric difference between two sets $A$ and $B: \Delta(A, B)$ is represented in white, the excluded part (the intersection of $A$ and $B$ ) is in black.

\section{Definition 6 (Nikodỳm distance).}

The Nikodym distance ([31]), denoted $d_{\Delta}$, is the Lebesgue measure ([22]) of the symmetric difference set of two (Lebesgue) measurable sets $A$ and $B\left(A, B \subset S\right.$ in $\left.\mathbb{R}^{n}\right)$ :

$$
d_{\Delta}(A, B)=\mathcal{L}^{n}(\Delta(A, B))
$$

where $\mathcal{L}^{n}$ denotes the Lebesgue measure in $\mathbb{R}^{n}$ (i.e. the area in $\mathbb{R}^{2}$ ).

The Steinhaus distance $([23,13])$ is derived from the symmetric difference set operation. It is defined by Eq. 12 for two Lebesgue measurable sets $A$ and $B$ in $\mathbb{R}^{n}$ with strictly positive measures.

Definition 7 (Steinhaus distance). The Steinhaus distance, denoted $d_{S}$, between two (Lebesgue) measurable sets $A$ and $B\left(A, B \subset S\right.$ in $\left.\mathbb{R}^{n}\right)$ is given by:

$$
d_{S}(A, B)=\frac{\mathcal{L}^{n}(\Delta(A, B))}{\mathcal{L}^{n}(A \cup B)}=1-\frac{\mathcal{L}^{n}(A \bigcap B)}{\mathcal{L}^{n}(A \bigcup B)}
$$

This distance may also be referenced as the Jaccard index ([19]).

\section{Geometric distances and spatial mosaics}

This section will show that the previous distances are not adapted to compare Jordan mosaics. Notice that a Jordan mosaic is being defined by its contours (see Def. 1). 
The Hausdorff distance may be applied on Jordan mosaics because they consist in compact sets. Since this distance uses a supremum operator, it is very sensitive to small spatial variations, as noted in [21]. A solution to this problem was proposed in [3] in the case of the Hausdorff distance, by replacing the supremum operator by a mean or p-th order mean operator. This distance is thus less sensitive to small spatial variations. In the case of discrete sets (i.e. sets in $\mathbb{Z}^{n}$ ), the sensitivity of the Hausdorff distance can be attenuated by taking the $\mathrm{k}$-th point that realises the supremum $([5])$, but the problem of sensitivity still persists.

\subsection{Nikodỳm and Steinhaus distances}

The Nikodỳm ans Steinhaus distances have an overwhelming drawback. They are not defined for Jordan mosaics, since these sets are not Lebesgue-measurable (a Jordan mosaic is indeed defined as a collection of contours, see Def. 1). They consequently cannot be used "as is" to compare Jordan mosaics.

\section{Extended geometric distances}

4.1 Minkowski addition and parallel neighborhoods

The Minkowski addition ([28]) defines an algebraic operation between sets in $\mathbb{R}^{n}$. It will be used to spatially enlarge the sets to be compared in order to be less sensitive to small spatial differences and/or to become Lebesgue measurable. Extensions of the Hausdorff distance and of the Nikodym distance will thus be introduced.

\section{Definition 8 (Minkowski addition).}

If $A$ and $B$ are two sets of $\mathbb{R}^{n}$, the Minkowski sum of $A$ and $B$, denoted $A \oplus B$, is then defined by:

$$
\begin{aligned}
& A \oplus B=\{a+b \mid a \in A, b \in B\} \\
& A \oplus B=\bigcup_{b \in B}\{a+b, a \in A\}
\end{aligned}
$$

where $\oplus$ is the Minkowski addition symbol.

The Hausdorff and the Nikodỳm distances are extended by introducing a spatial enlargement defined by the mean of the Minkowski addition.

Definition 9 (Unit neighborhood).

$N \in \mathbb{R}^{n}$ denotes the unit neighbourhood (e.g., the $\mathrm{n}$-dimensional unit ball in $\mathbb{R}^{n}$, Def. 9). The point $O$ is the origin of $\mathbb{R}^{n}$.

$$
N=\left\{x \in \mathbb{R}^{n}, d_{E}(O, x) \leq 1\right\}
$$

With this notation, $r N$ designates the ball of radius $r$, centered on the origin $O$.

Definition 10 (r-enlargement). The closed $r$-enlargement of a set $A$ in $\mathbb{R}^{n}$ is defined as:

$$
A_{r}=A \oplus r N
$$

$A_{r}$ denotes the $r$-enlarged set of $A$.

$A_{r}$ is also classically called the parallel set at distance $r$ of $A$ or simply the $r$-parallel set to $A$ ([38,44]). It has also been called the tubular neighborhood in [16]. The effect of the Minkowski addition can be seen on Fig. 5 . 


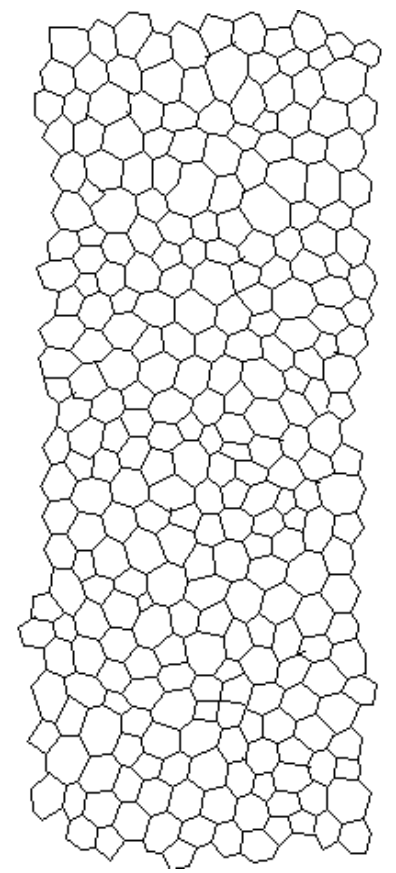

1. Reference Jordan mosaic $(M)$.

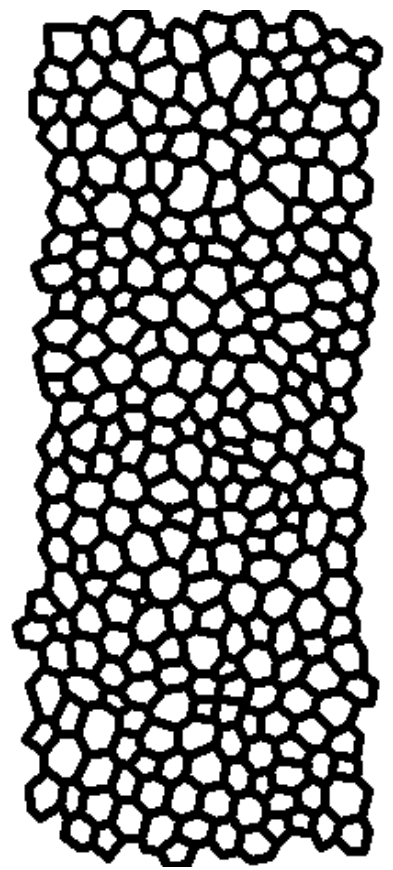

2. Enlargement of $M$ for a parameter value $r=3$ (dilation $M \oplus r N)$.

Fig. 5 Enlargement (Subfig. 2.) of the reference mosaic $M$ (Subfig. 1.).

\subsection{Extended Hausdorff distance}

\section{Definition 11 (Extended Hausdorff distance).}

Let $r \in \mathbb{R}(r \geq 0)$ be a positive real number. The extended Hausdorff distance is defined for two compact sets $A$ and $B$, using the unit neighborhood $N$ :

$$
\begin{array}{r}
d_{H}^{r}(A, B)= \\
\max \left\{f_{d_{E}}(A \oplus r N, B), f_{d_{E}}(B \oplus r N, A)\right\}
\end{array}
$$

The Hausdorff distance can also be defined by using the Minkowski addition $\oplus$ (see Sect. 4.1):

$$
d_{H}^{r}(A, B)=\max \left\{f_{d_{E}}^{r}(A, B), f_{d_{E}}^{r}(B, A)\right\}
$$

with

$$
f_{d_{E}}^{r}(A, B)=\inf \{p \in \mathbb{R} \mid(B \oplus r N) \subset(A \oplus p N)\}
$$

Proposition 1 The half-distance between $A$ and $B$ (Eq. 7) is the minimal value of $p$ so that the dilation of $A$ (which is $A \oplus p N$ ) englobes the set $B$ :

$$
f_{d_{E}}(A, B)=\inf \{p \in \mathbb{R}, B \subset(A \oplus p N)\}
$$

This formulation implies the next relation (Eq. 15), which means that there is an almost linear relation between the Hausdorff distance and its extension, namely:

$$
\begin{array}{cc}
f_{d_{E}}^{r}(A, B) & =\quad \inf \{p \in \mathbb{R} \mid \\
& B \subset(A \oplus(\max \{p-r ; 0\}) N)\} \\
d_{H}^{r}(A, B)= & \max \left\{d_{H}(A, B)-r ; 0\right\}
\end{array}
$$

Thus, if the Hausdorff distance is sensitive to small variations, this is also the case for its extension. This is why it is preferable not to use the extended Hausdorff distance for Jordan mosaic comparison purpose. 
4.3 Extended Nikodỳm distance

\section{Definition 12 (Extended symmetric difference).}

The extension of the symmetric difference, denoted $\Delta^{r}$, is the symmetric difference involving a $r$ enlargement, defined by:

$$
\Delta^{r}(A, B)=\left(A \backslash B_{r}\right) \cup\left(B \backslash A_{r}\right)
$$

This notation introduces an enlargement in the symmetric difference. Notice that this tolerance $r$ would not exist when considering the operations $\left(A_{r} \backslash B_{r}\right)$ and $\left(B_{r} \backslash A_{r}\right)$.

Definition 13 (Extended Nikodỳm distance).

The extended Nikodỳm distance $d_{\Delta^{r}}$ is then defined for two Lebesgue measurable sets $A$ and $B$ in $\mathbb{R}^{n}$ by:

$$
d_{\Delta^{r}}(A, B)=\mathcal{L}^{n}\left\{\Delta^{r}(A, B)\right\}
$$

Proposition 2 The extended Nikodym distance does not verify the axiom of separation and the triangle inequality (Eqs. 4 and 6, respectively) of a metric (because of the tolerance parameter $r$ ). It verifies the symmetry axiom (Eq. 5).

4.4 Distances are not adapted for visual comparison

A distance is the mathematical tool classicaly used for sets comparison. In the case of the Hausdorff and Nikodym distances, it appears that small spatial variations or the lack of Lebesgue measurability make them inefficient or not available. Thus, a notion of geometric enlargement is introduced, but loosing the properties of a distance (the separation axiom and the triangle inequality are not satisfied). The question of the relevance of the distance notion is therefore clearly stated. It will be now discussed in the context of the human visual perception.

\section{Distances and human visual perception}

In this section, it will be highlighted that the axioms defining a metric are in fact not relevant to mathematically quantify the geometric differences that are visually percepted.

The following subsections will explain the non relevance of each metric axiom. Therefore, a comparison criterion cannot be defined as being a distance fonction with regards to the human visual perception.

\subsection{The separation axiom}

The human visual perception does not always consider that two distinct geometric objects (considered as closed bounded sets) are different. This means that a comparison criterion (denoted $c$ ) does not verify the separation axiom for two closed bounded sets $A$ and $B$ (Eq. 4):

$$
c(A, B)=0 \nRightarrow A=B
$$

This is what is called a visual tolerance: not identical sets $(\exists x \in A, x \notin B$ ) can be considered equal for the given criterion $c$.

\subsection{The triangle inequality axiom}

It has been proved that the triangle inequality is not respected by the human visual perception system ([41, 43, 42]) as illustrated in Fig. 6.

Mathematically, this means that for a given comparison criterion $c$ and two closed bounded sets $A$ and $B$, the triangle inequality (Eq. 6) is not respected, namely:

$$
\exists z \mid c(A, B)>c(A, C)+c(C, B)
$$




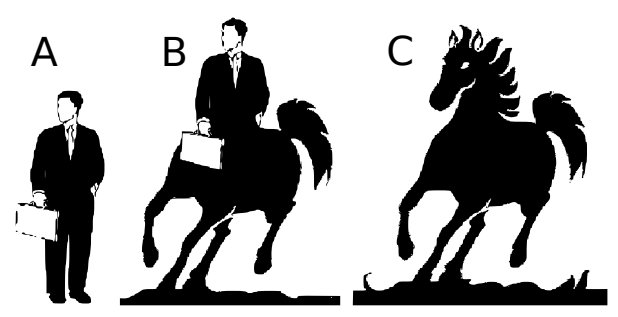

Fig. 6 The triangle inequality is not verified by the visual perception. The horse and the man are really different, but the distance between the man and the centaur (or between the centaur and the horse) may appear small. This means $c(A, C)>c(A, B)+c(B, C)$

\subsection{The symmetry axiom}

More surprisingly, the visual perception system does not satisfy to the symmetry principle ([40]).

Mathematically, the symmetry (Eq. 5) is not respected for a given comparison criterion $c$, namely:

$$
c(A, B) \neq c(B, A)
$$

In the case of an application issue where a reference geometric object is considered, the loss of this axiom can become relevant.

\subsection{Dissimilarities}

It thus follows from the three previous subsections that the metric notion appears as not adapted to mimic the human visual perception system when comparing two geometric objects. Since the metric concept is too strong, the notion of dissimilarity has been proposed by psychologists $([41,37])$.

\section{Definition 14 (Dissimilarity).}

Formally, a dissimilarity can be defined as a function $\varsigma$ of two objects $A$ and $B$, that verifies ([13])

$$
0 \leq \varsigma(A, A) \leq \varsigma(A, B)
$$

This means that the higher $\varsigma(A, B)$ is, the more dissimilar $A$ and $B$ are.

Tversky ([40]) proposes the notion of dissimilarity function $\varsigma$ between two sets $A$ and $B$ (Def. 15). Notice that a dissimilarity is dissymmetric by construction.

\section{Definition 15 (Dissimilarity function).}

A dissimilarity function between two sets $A$ and $B$, denoted $\varsigma(A, B)$, can be expressed by:

$$
\varsigma(A, B)=\theta f(A \cap B)-\beta f(A \backslash B)-\gamma f(B \backslash A)
$$

where $\theta, \beta$ and $\gamma$ are real numbers and $f$ is a measuring function ([40]).

\section{A geometric dissimilarity criterion for mosaics}

Let $M, X, Y$, and $Z$ be Jordan mosaics defined through their contours collections. $M$ will designate the reference mosaic and $X, Y, Z$ will designate the mosaics compared to $M$. Remember that a Jordan mosaic is thus not Lebesgue-measurable. 


\subsection{Definitions}

To deal with the problem of the non-Lebesgue measurability of a Jordan mosaic $M$, a solution is to use the enlarged mosaic $\left.M_{r}\right|_{S}=\left.(M \oplus r N)\right|_{S}$ instead of $M$ in the symmetric difference definition (Eq. 10). To simplify the notations, $M_{r}$ will denote $\left.M_{r}\right|_{S}$, the $r$-enlarged mosaic $M$ constrained to the domain $S$. Using the Minkoswki addition for $r>0$, the enlarged mosaic $M_{r}$ is Lebesgue-measurable.

\section{Definition 16 (Enlarged mosaic).}

Let $r$ be a strictly positive real number. An $r$-enlarged Jordan mosaic $M_{r}$ is defined by the tubular $r$ neighborhood of the Jordan mosaic $M$ (see Def. 10):

$$
M_{r}=M \oplus r N
$$

Definition 17 (Extension and tolerance parameters).

For a $r$-enlarged Jordan mosaic, $r$ is called the enlargement parameter.

- It will be called the tolerance parameter and denoted $\rho$ when the goal is to involve a $(r$-level) of spatial variations.

- It will be called the extension parameter and denoted $\alpha$ in the cases where the Lebesgue measurability is required.

6.2 The $\epsilon$ dissimilarity criterion

\section{Definition 18 (Dissimilarity criterion)}

The dissimilarity criterion $\epsilon$, depending on $\rho$ (tolerance parameter), $\alpha$ (extension parameter) and $M$ (reference Jordan mosaic), and based on the extended symmetric difference $\Delta^{\rho}$, compares a Jordan mosaic $X$ to the reference Jordan mosaic $M$ :

$$
\begin{aligned}
\epsilon_{M}^{\rho, \alpha}(X) & =\frac{d_{\Delta^{\rho}\left(M_{\alpha}, X_{\alpha}\right)}}{\mathcal{L}^{n}\left\{M_{\alpha} \oplus \rho N\right\}} \\
& =\frac{\mathcal{L}^{n}\left\{\left(X_{\alpha} \backslash M_{\alpha} \oplus \rho N\right) \cup\left(M_{\alpha} \backslash X_{\alpha} \oplus \rho N\right)\right\}}{\mathcal{L}^{n}\left\{M_{\alpha} \oplus \rho N\right\}}
\end{aligned}
$$

\section{Proposition 3 ( $\epsilon$ is a dissimilarity function).}

The $\epsilon$ dissimilarity criterion (Def. 18) is a dissimilarity function.

Proof According to Def. 18, the dissimilarity criterion is given by:

$$
\epsilon_{M}^{\rho, \alpha}(X)=\frac{\mathcal{L}^{n}\left\{\left(X_{\alpha} \backslash M_{\alpha+r}\right) \cup\left(M_{\alpha} \backslash X_{\alpha+\rho}\right)\right\}}{\mathcal{L}^{n}\left\{M_{\alpha+\rho}\right\}}
$$

Since $\left(X_{\alpha} \backslash M_{\alpha+\rho}\right) \cap\left(M_{\alpha} \backslash X_{\alpha+\rho}\right)=\emptyset$, this implies:

$$
\epsilon_{M}^{\rho, \alpha}(X)=\frac{\mathcal{L}^{n}\left\{\left(X_{\alpha} \backslash M_{\alpha+\rho}\right)\right\}+\mathcal{L}^{n}\left\{\left(M_{\alpha} \backslash X_{\alpha+\rho}\right)\right\}}{\mathcal{L}^{n}\left\{M_{\alpha+\rho}\right\}}
$$

This relation proves that $\epsilon$ is a dissimilarity criterion as defined by [40] (Def. 15), with $\beta=\gamma=-1, \theta=0$ and $f$ designates the Lebesgue measure $\mathcal{L}^{n}$.

\subsection{Properties}

\section{Proposition 4 (Symmetry).}

The $\epsilon$ dissimilarity criterion does not verify the symmetry axiom of a metric.

$$
\epsilon_{M}^{\rho, \alpha}(X) \neq \epsilon_{X}^{\rho, \alpha}(M)
$$

This is because the purpose of this criterion is to compare a Jordan mosaic to a given reference Jordan mosaic. This dissymmetry expresses that the two mosaics $M$ and $X$ do not play the same role, $M$ being the reference Jordan mosaic considered to be the truth (up to a tolerance value $\rho$ ) to which the Jordan mosaic $X$ is to be compared. 


\section{Proposition 5 (Positivity).}

The $\epsilon$ dissimilarity criterion is positively valued:

$$
\forall X \subset S, \epsilon_{M}^{\rho, \alpha}(X) \geq 0
$$

Proposition 6 (Identity).

The identity axiom (Eq. 3) is verified:

$$
\epsilon_{M}^{\rho, \alpha}(M)=0
$$

Proposition 7 (Separation).

The separation axiom (Eq. 4) is not verified by definition:

$$
\epsilon_{M}^{\rho, \alpha}(X)=0 \Rightarrow\left\{\begin{array}{l}
X \subset X_{\alpha} \subset M \oplus \rho N \\
M \subset M_{\alpha} \subset X \oplus \rho N
\end{array}\right.
$$

In the general case, $X \neq M$. This is explained by the fact that the tolerance parameter $\rho$ makes two distinct (but close) Jordan mosaics as equal.

\section{Proposition 8 (Triangle inequality).}

The triangle inequality (see Eq. 6) is not verified.

Proof For example, $X, Y$ and $Z$ being chosen as illustrated in Fig. 7:

$$
\begin{aligned}
& d_{\Delta^{\rho}}(X, Z)=0 \\
& d_{\Delta^{\rho}}(Z, Y)=0
\end{aligned}
$$

It is possible to choose $X$ and $Y$ so that $d_{\Delta^{\rho}}(X, Y)>0$, and thus the inequality $d_{\Delta^{\rho}}(X, Y) \leq d_{\Delta^{\rho}}(X, Z)+$ $d_{\Delta^{\rho}}(Z, Y)$ is not verified (see Figure Fig. 7).

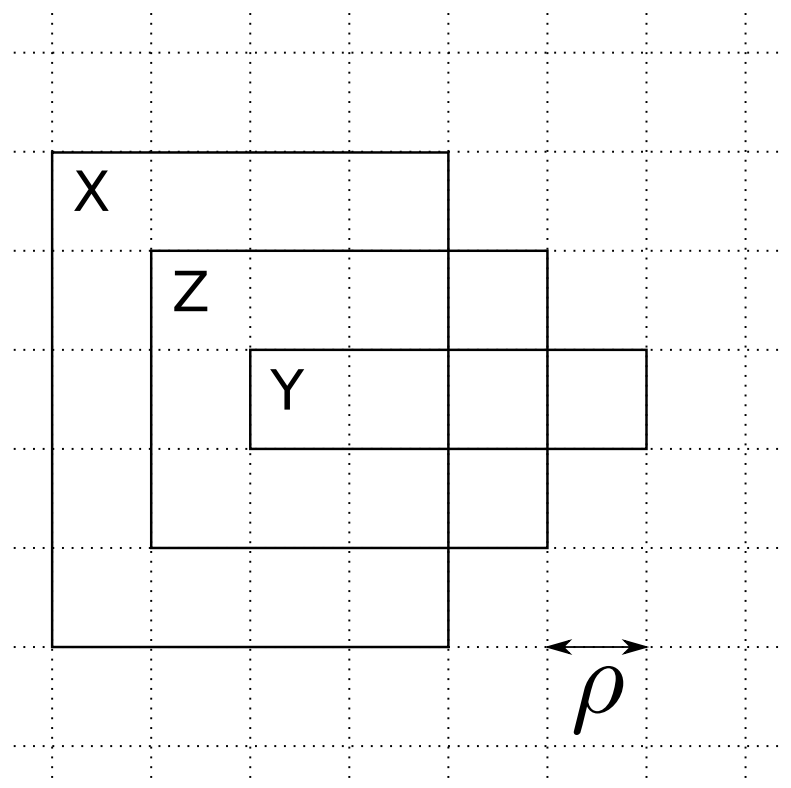

Fig. 7 The triangle inequality is not verified in this case (see the value of $\rho$ ). Each layer line is at a distance of $\rho$ of the next one. $d_{\Delta^{\rho}}(X, Z)=0$ and $d_{\Delta^{\rho}}(Z, Y)=0$, but $d_{\Delta^{\rho}}(X, Y)>0$ 


\section{Properties of the $\epsilon$ dissimilarity criterion: general case $\rho>0, \alpha>0$}

\subsection{Properties}

\section{Proposition 9 (Unboundedness).}

The value given by the $\epsilon$ dissimilarity criterion is not bounded.

Proposition 10 (Monotonicity).

The $\epsilon$ dissimilarity criterion is decreasing in regard to the tolerance parameter $\rho$.

$$
\forall\left(\rho_{1}, \rho_{2}\right) \in \mathbb{R}_{+}^{2}, \rho_{1}>\rho_{2} \Rightarrow \epsilon_{M}^{\rho_{1}, \alpha} \leq \epsilon_{M}^{\rho_{2}, \alpha}
$$

Proof If a greater tolerance value is taken, the $\epsilon$ value will decrease because the following relations hold:

$$
\begin{aligned}
\Delta_{M}^{\rho_{2}}(X) & \subset \Delta_{M}^{\rho_{1}}(X) \\
\mathcal{L}^{n}\left(M \oplus \rho_{2} N\right) & <\mathcal{L}^{n}\left(M \oplus \rho_{1} N\right)
\end{aligned}
$$

7.2 Asymptotic behavior and geometric invariances

Theorem 1 (Asymptotic behavior).

Beyond a certain tolerance value $\rho_{0}$, the $\epsilon$ dissimilarity criterion values equal zero. This value $\rho_{0}$ is the Hausdorff distance between $X$ and $M$.

$$
\begin{array}{r}
\forall X \subset S, \exists \rho_{0} \mid \forall \rho \geq \rho_{0}, \epsilon_{M}^{\rho}(X)=0 \\
\text { where } \rho_{0}=d_{H}(X, M)
\end{array}
$$

Proof It can easily be proved with Eq. 13 and Prop. 7.

Proposition 11 (Scale invariance).

The $\epsilon$ dissimilarity criterion remains invariant through an homothetic transformation:

$$
\forall \lambda \in \mathbb{R}_{+}, \epsilon_{\lambda M}^{\lambda \rho, \alpha}(\lambda X)=\epsilon_{M}^{\rho, \alpha}(X)
$$

Proof Let's start by the definition of the extended symmetric difference (Def. 12).

$$
\Delta^{\rho}(X, Y)=(X \backslash Y \oplus \rho N) \cup(Y \backslash X \oplus \rho N)
$$

Thus, the Lebesgue measure of the symmetric difference is:

$$
\begin{aligned}
d_{\Delta^{\rho}}\left(X_{\alpha}, Y_{\alpha}\right)= & \mathcal{L}^{n}\left(\Delta^{\rho}\left(X_{\alpha}, Y_{\alpha}\right)\right) \\
= & \mathcal{L}^{n}\left(X_{\alpha} \backslash Y_{\alpha} \oplus \rho N\right) \\
& +\mathcal{L}^{n}\left(Y_{\alpha} \backslash X_{\alpha} \oplus \rho N\right)
\end{aligned}
$$

When multiplying $X$ and $Y$ by a scalar $\lambda$ (notice that the relation $\lambda(A \oplus B)=\lambda A \oplus \lambda B$ is verified), yields:

which finally gives:

$$
\begin{array}{r}
d_{\Delta^{\rho}}\left(\lambda X_{\alpha}, \lambda Y_{\alpha}\right)= \\
\lambda^{n} \mathcal{L}^{n}\left(X_{\alpha} \backslash Y_{\alpha} \oplus \frac{\rho}{\lambda} N\right)+\lambda^{n} \mathcal{L}^{n}\left(Y_{\alpha} \backslash X_{\alpha} \oplus \frac{\rho}{\lambda} N\right)
\end{array}
$$

$$
d_{\Delta^{\rho}}\left(\lambda X_{\alpha}, \lambda Y_{\alpha}\right)=\lambda^{n} d_{\Delta \frac{\rho}{\lambda}}\left(X_{\alpha}, Y_{\alpha}\right)
$$

In other words, a change of scale for both the considered Jordan mosaic and the reference Jordan mosaic gives allways the same $\epsilon$ dissimilarity value for a scaled tolerance $\lambda \rho$.

Proposition 12 (Invariance by rigid geometric transformation).

If $\tau$ is a rigid geometric transformation (like translation, rotation, reflection or symmetry), the following relation is true:

$$
\epsilon_{\tau(M)}^{\rho, \alpha}(\tau(X))=\epsilon_{M}^{\rho}(X)
$$

This means that applying the same rigid transformation to two Jordan mosaics yields to the same $\epsilon$ dissimilarity value. 


\section{Properties of the $\epsilon$ dissimilarity criterion: role of the $\alpha$ extension parameter}

The $\alpha$ extension parameter guarantees the Lebesgue measurability of the considered $\alpha$-enlarged Jordan mosaics $X_{\alpha}$ and $M_{\alpha}$. In the general case with a tolerance $\rho>0$, the dissimilarity criterion $\epsilon$ is defined for all values of $\alpha$ (i.e. even for $\alpha=0$, see Def. 18).

\subsection{Definitions}

Definition 19 (for $\alpha=0$ and $\rho>0$ ).

For $\alpha=0$ and $\rho>0, \epsilon_{M}^{\rho, \alpha=0}(X)=0$. This is due to the non Lebesgue measurability of $X$ and $M$.

Definition 20 (for $\alpha>0$ and $\rho=0$ ).

For $\alpha>0, \epsilon_{M}^{\rho=0, \alpha}(X)$ is perfectly defined (see Def. 18)).

The question arises when $\alpha$ tends toward 0 .

8.2 Limit for $\alpha \rightarrow 0, \rho=0$

The symbol $\mathcal{L}^{n}$ will still denote the Lebesgue measure in $\mathbb{R}^{n}$ (i.e. the area in $\mathbb{R}^{2}$ ), while the symbol $\mathcal{H}^{n}$ will denote the Hausdorff measure in $\mathbb{R}^{n}$ ([29]).

In order to study this asymptotic behavior, it is necessary to introduce the so-called Minkowski content:

Definition 21 (Minkowski content).

The Minkowski content ([15]) of a finite union of (n-1)-dimensional compact Lipschitz surfaces $A \in \mathbb{R}^{n}$, denoted $\mathcal{M}^{n-1}(A)$, is defined as follows:

$$
\mathcal{M}^{n-1}(A)=\lim _{\alpha \rightarrow 0} \frac{\mathcal{H}^{n}\left(A_{\alpha}\right)}{2 \alpha}
$$

The discussion and study of the existence conditions of the limit (Def. 21) are outside the scope of the present article. The reader can refer to $[2,44]$ for recent advances. A nice condition is fullfilled ([2]) for

- a Jordan mosaic $A$ which consists of finite union of Lipschitz $(n-1)$-dimensional surfaces

- and a finite union or intersection of such Jordan mosaics ([29]).

\section{Definition 22 (Lipschitz mosaic).}

A (finite) Lipschitz mosaic is a (finite) Jordan mosaic that consists of a (finite) union of Lipschitz surfaces.

This is a condition that is practically obtained in the case of real physical mosaics $([17,9,27])$.

The following proposition straightforwardly based on [15] will be of a great interest in the Sect. 8.3.

\section{Proposition 13 (Minkowski content and}

Hausdorff measures).

The Minkowski content coincides with the $(n-1)$-dimensional Hausdorff measure, namely:

$$
\mathcal{M}^{n-1}(X)=\mathcal{H}^{n-1}(X)
$$

for $X$ a finite Lipschitz mosaic, and a finite union or intersection of Lipschitz mosaics. 


\subsection{Asymptotic behavior}

\section{Theorem 2 (Limit of extension).}

Let $X$ and $M$ be two Lipschitz mosaics. The dissimilarity criterion $\epsilon_{M}^{\rho=0, \alpha}$ for $\rho=0$ is defined when $\alpha \rightarrow 0$ by:

$$
\lim _{\alpha \rightarrow 0} \epsilon_{M}^{\rho=0, \alpha}(X)=\frac{\mathcal{H}^{n-1}(\Delta(M, X))}{\mathcal{H}^{n-1}(M)}
$$

Proof Starting with:

$$
\epsilon_{M}^{\rho=0, \alpha}(X)=\frac{\mathcal{L}^{n}\left\{\left(X_{\alpha} \cup M_{\alpha}\right) \backslash\left(X_{\alpha} \cap M_{\alpha}\right)\right\}}{\mathcal{L}^{n}\left\{M_{\alpha}\right\}}
$$

and by dividing both the numerator and denominator by $2 \alpha$ yields:

$$
\epsilon_{M}^{\rho=0, \alpha}(X)=\underbrace{\frac{\frac{\mathcal{L}^{n}\left(X_{\alpha} \cup M_{\alpha}\right)}{2 \alpha}}{\frac{\mathcal{L}^{n}\left(M_{\alpha}\right)}{2 \alpha}}}_{\epsilon_{1}}-\underbrace{\frac{\frac{\mathcal{L}^{n}\left(X_{\alpha} \cap M_{\alpha}\right)}{2 \alpha}}{\frac{\mathcal{L}^{n}\left(M_{\alpha}\right)}{2 \alpha}}}_{\epsilon_{2}}
$$

First, it will be shown that $\epsilon_{1}$ and $\epsilon_{2}$ (see Eq. 17) have finite limits for $\alpha \rightarrow 0$, prooving that $\lim \left(\epsilon_{1}-\epsilon_{2}\right)=$ $\lim \left(\epsilon_{1}\right)-\lim \left(\epsilon_{2}\right)$.

As the union and dilation operators are continuous and by using Def. 21 and Prop. 13, yields:

$$
\lim _{\alpha \rightarrow 0} \epsilon_{1}=\frac{\mathcal{H}^{n-1}(X \cup M)}{\mathcal{H}^{n-1}(M)}
$$

As the intersection operator is upper semi-continuous and as the dilation operator is continuous, yields:

$$
\limsup _{\alpha \rightarrow 0}\left(X_{\alpha} \cap M_{\alpha}\right)=X \cap M
$$

and

$$
\lim _{\alpha \rightarrow 0}(X \cap M)_{\alpha}=X \cap M
$$
that:

In addition, the nesting property of the $\left(X_{\alpha} \cap M_{\alpha}\right)$ family (i.e. $X_{\alpha} \cap M_{\alpha} \subset X_{\alpha^{\prime}} \cap M_{\alpha^{\prime}}$, for $\left.\alpha<\alpha^{\prime}\right)$ implies

$$
\liminf _{\alpha \rightarrow 0}\left(X_{\alpha} \cap M_{\alpha}\right)=X \cap M
$$

Therefore, and by using Def. 21 and 13:

$$
\lim _{\alpha \rightarrow 0} \epsilon_{2}=\frac{\mathcal{H}^{n-1}(X \cap M)}{\mathcal{H}^{n-1}(M)}
$$

Theorem 2 is thus established.

This theorem leads to the following definition:

Definition 23 (for $\alpha=0$ and $\rho=0$ ).

The $\epsilon$ dissimilarity criterion with no tolerance and with no enlargement is defined by:

$$
\epsilon_{M}^{\rho=0, \alpha=0}(X)=\frac{\mathcal{H}^{n-1}(\Delta(M, X))}{\mathcal{H}^{n-1}(M)}
$$

For example, in $\mathbb{R}^{2}$, this value is the length of the symmetric difference divided by the length of the reference mosaic. 


\subsection{Discussion}

Therefore, for Lipschitz mosaics $X$ and $M$ (consisting of finite union of $(n-1)$ dimensional Lipschitz surfaces) the geometric dissimilarity criterion $\epsilon$ with no geometric tolerance $(\rho=0)$ and no geometric extension $(\alpha=0)$ is given by Def. 23 as a limit value. It is equal to the ratio of the surface area not shared by the Lipschitz mosaics $X$ and $M$ divided by the surface area of the reference Lipschitz mosaic $M$. This result corresponds to what is intuitively infered in the 2-dimensional case when looking at a plane figure. In practical situations Def. 23 is not directly calculable and Def. 18 is therefore the relevant definition for the geometric dissimilarity criterion $\epsilon$. This is of great importance from a practical viewpoint. This was also theoretically important to establish that Theorem 2 gives the geometric limit case when passing from the $n$-dimensional to the $(n-1)$-dimensional case for the $\epsilon$ dissimilarity criterion.

\section{Conclusion}

This first part introduced the notion of Jordan mosaics as a finite union set of hypersurfaces in $\mathbb{R}^{n}$. The goal was to compare Jordan mosaics to a reference Jordan mosaic. It showed that classical distances (Hausdorff, Nikodym...) are not adapted for these special sets. A novel criterion has been proposed, which appeared to be in accordance with the notion of dissimilarity proposed by psychologists and that better suits what the human visual perception can perform. It possesses strong properties such as monotonicity and invariance under scaling or rigid geometric transformations. Its asymptotic behavior is also established for a special case of Jordan mosaics called Lipschitz mosaics.

\section{Part II: Application and comparison issues}

\section{Introduction}

The first part of this article introduced a dissimilarity criterion adapted to Jordan mosaics, called the $\epsilon$ dissimilarity criterion. From an image analysis point of view, the result of an image segmentation process can often be seen as a Jordan spatial mosaic. There are two different ways of presenting a result of a segmentation, either region-based or contour-based approach. According to [24], the human visual perception first rely on contours; thisjustifies the fact that the mosaics are some sets of contours and that the $\epsilon$ criterion evaluates the dissimilarities between these contours. The possibility of comparing region-based segmentation results will not be discussed.

This second part practically illustrates the properties of this criterion in the case of real segmented images of human corneal endothelium. It also proposes a way to choose the value of the tolerance parameter $\rho$. Thus, the considered mosaic is defined by the contours of the endothelial cells. Some dissimilarity criteria found in the segmentation evaluation litterature are afterwards presented. Next, this paper proposes to evaluate the performance of these criteria in regard to some transformations (over and under segmentations, translations, distortions and small spatial variations.).

\subsection{Discrete case}

This second part of the article presents the results in the case of real binary images, i.e. for discrete sets. Thus, the following notations are introduced: $I_{M}$ and $I_{X}$ represent binary images that correspond to a reference mosaic $M$ and another mosaic $X$. An image segmentation result can be seen from a contour point of view as a special mosaic defined with a non null extension parameter $\alpha$. In this case, a mosaic is a binary image of the contours. There will be no further mention of the extension parameter $\alpha$ because it is already involved in the segmentation result.

Then, the $\epsilon$ dissimilarity criterion applied to binary images is defined by the following equation:

$$
\epsilon_{M}^{\rho}(X)=\frac{\#\{(X \backslash M \oplus \rho N) \cup(M \backslash X \oplus \rho N)\}}{\#\{M \oplus \rho N\}}
$$

with $N$ being the structuring element of radius 1 ( $\rho N$ is the discrete ball of radius $\rho$ ) and \# meaning the number of non null pixels in the set (cardinal operator) (see Fig. 5). 
A database of 30 different mosaics is used and for each one, an expert ophthalmologist has manually segmented the cells and thus created a reference mosaic (Fig. 25) for each image (Fig. 26). The Fig. 8 illustrates the properties of monotonicity (Prop. 10) and the asymptotic behavior (Theorem 1).

\subsection{Choice of the tolerance parameter value}

This subsection explains how to fix the tolerance parameter $\rho$ according to the considered mosaics. With the image database (Fig. 26), the experts have 10 times drawn the same mosaic, and the $\epsilon$ dissimilarity criterion have been used to compare every manual segmentation to the others. The mean value of the $\epsilon$ dissimilarity criterion is represented in the Fig. 9. The reader can consider that an expert will always draw the contours at the same location within a certain spatial tolerance (i.e. within the tolerance tube), depending on the image size and the precision of the drawing tool.

In the future, the choice of the tolerance parameter could be made automatically by an adaptive approach, depending on the mosaic itself, by defining this parameter as (for example) a function of the curvature [30,20] or by using some greyscale informations [12]. Thus, this parameter would be independant of any supervision.

For this database, the tolerance parameter value is $\rho=2$.

\section{Evaluation criteria}

Evaluating the results of image segmentation methods is not a recent subject, but it still remains a difficult task.

A segmentation process, in the field of image analysis, provides a partition of the spatial definition domain $S$ of the gray tone images into adjacent regions that present a particular interest. It is often useful to quantify the performance of such a segmentation process: numerous methods exist for this task $([50,10])$.

Evaluation methods can be divided into different categories $([33,49])$. Supervised methods are based on the comparison versus an expert, which gives a reference partition of a domain $S$. Unsupervised methods consist on establishing an absolute criterion based on some already known characteristics (for example, homogeneity of luminance, contrast...). They are also called stand-alone evaluations. The supervised methods are very time-consuming because they require experts to give their own solutions, often manually drawn.

When choosing between one of these two types of methods, the key question of subjectivity is raised. Often, when a human expert gives his own result of segmentation, it may be slightly different from another expert. This is why, when evaluating segmentation methods, unsupervised criteria are often preferred. But on the other way, unsupervised methods are based on specific characteristics, and the choice of these characteristics can introduce a bias in the comparison: the subjectivity is therefore also present in these methods.

In our point of view, supervised methods are to be preferred instead of unsupervised methods, because we consider that it is better to take into account that there is a variability in the expertise instead of believing that an unsupervised method would be unbiased.

\section{Presentation of 11 evaluation criteria}

The following pages will consider a criterion crit comparing two mosaics $M$ and $X$, where $M$ is the reference mosaic (from the expert). It will be denoted $\operatorname{crit}_{M}(X)$. The Euclidean distance $d$ will also be mentionned, and the notation $d(p, M)$ is the distance between a pixel $p$ and a mosaic $M . \mathcal{N}$ is now the size of the images $I_{M}$ or $I_{X}$, considered as equal for obvious practical reasons.

\subsection{Dissimilarity criteria}

Remember psychologists proposed the notion of dissimilarity (15):

$$
0 \leq \varsigma(A, A) \leq \varsigma(A, B)
$$

Notice that the 11 presented criteria are dissimilarity criteria. 
Monotonicity of the $\epsilon$ criterion

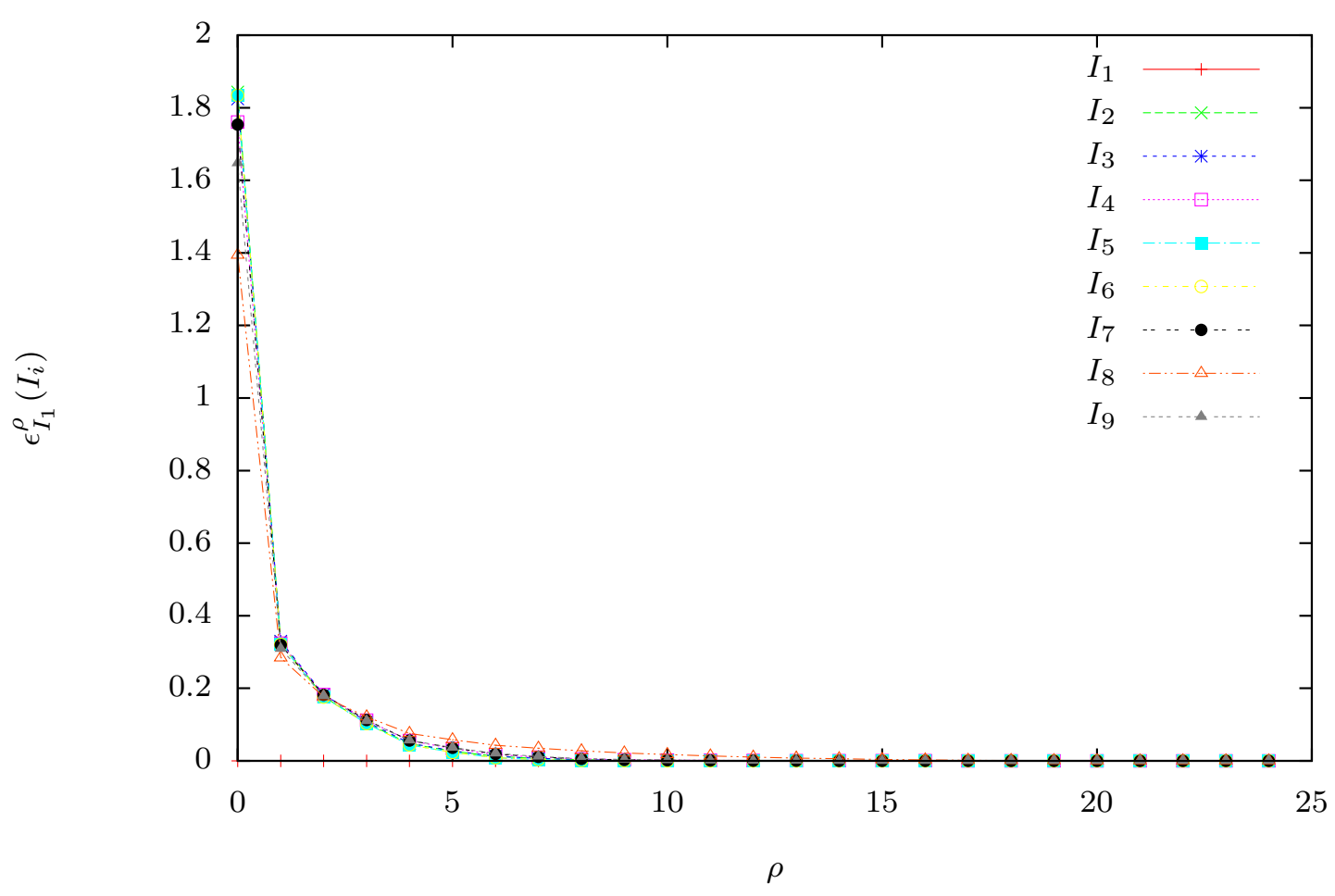

1. Log scale.

Monotonicity of the $\epsilon$ criterion

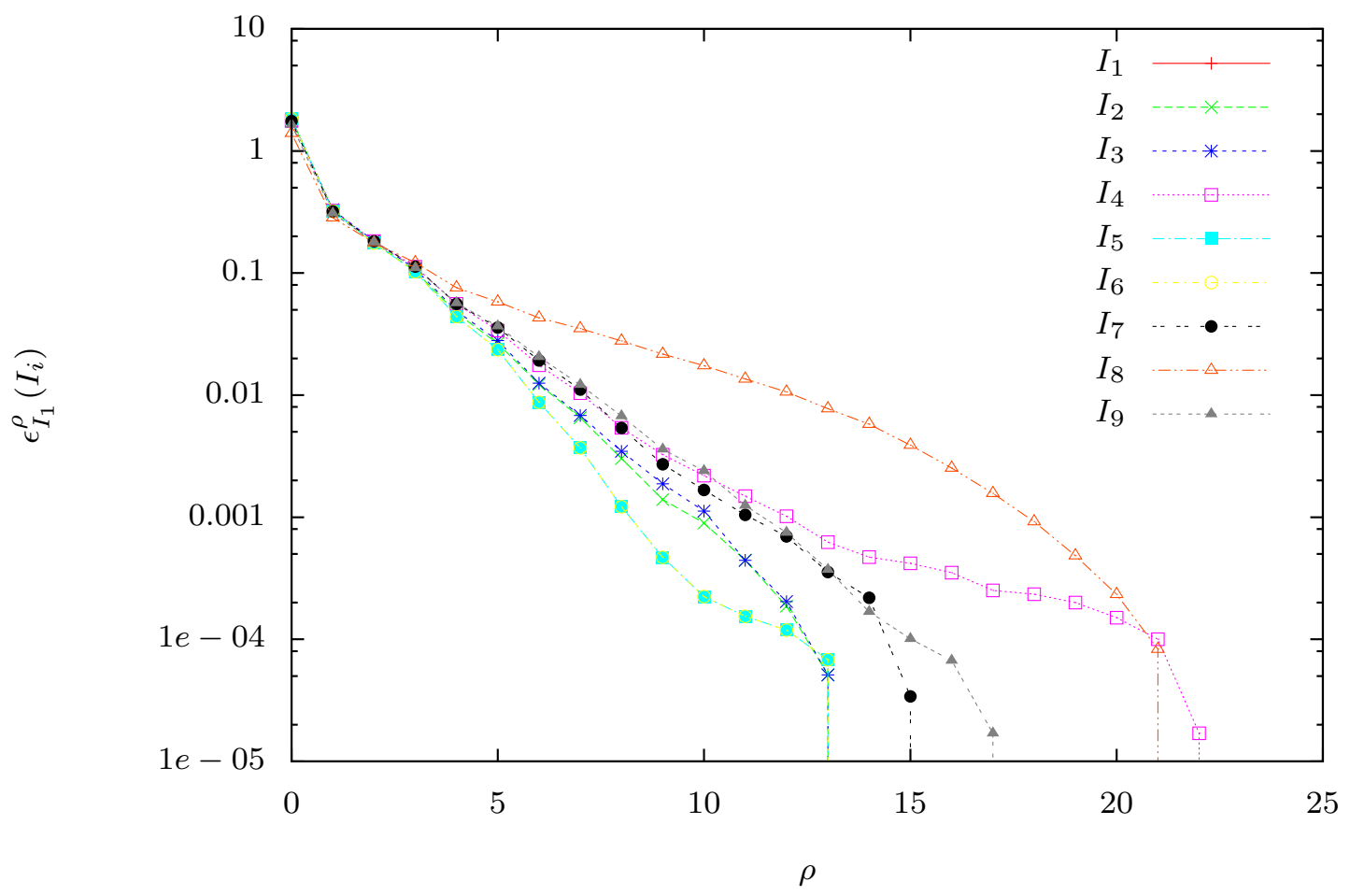

2. Normal scale.

Fig. 8 This graph represents the evolution of $\epsilon_{I_{1}}^{\rho}\left(I_{i}\right)$ (in logscale), with $i=1 . .9$ and $I_{i}$ representing segmentations of different images. When the tolerance parameter $\rho$ increases, the dissimilarity value decreases and is null after some value (that differs for each image $I_{i}$ ), the vertical line is due to the logscale. 
Errors made by an expert ophtalmologist

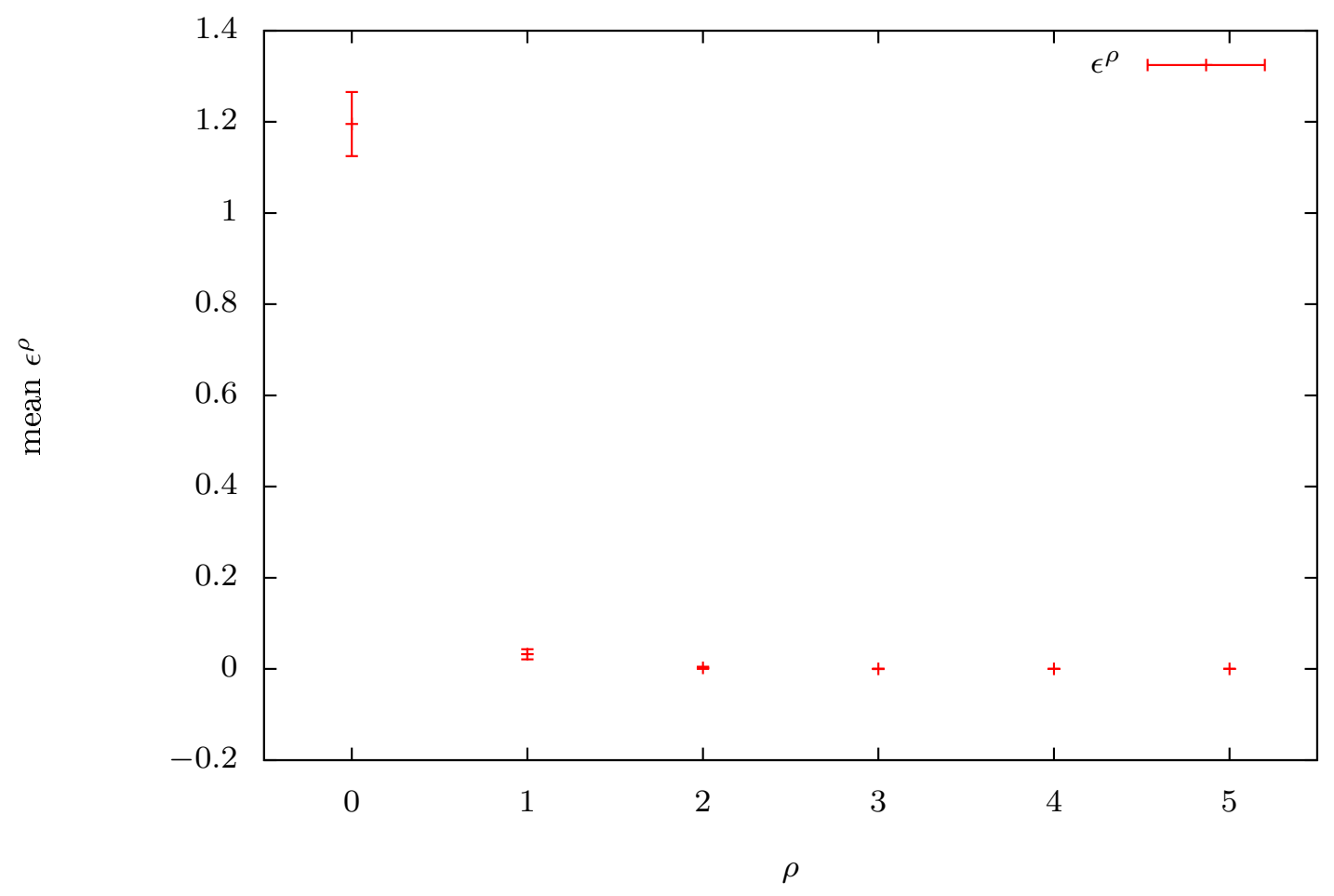

Fig. 9 Method for fixing the tolerance parameter. In this example, $\rho$ is in pixels, and there is a strong gap between no tolerance $(\rho=0)$ and a tolerance of one pixel $(\rho=1)$.

12.211 dissimilarity criteria

The criteria are separated into three main categories:

- the criteria that use the Euclidean distance between pixels,

- the criteria based on the symmetric difference of sets,

- the criteria based on pixel-to-pixel operations, including their neighbors.

\subsection{Euclidean distance between pixels and derivatives}

In the case of mosaics comparisons, the Hausdorff distance haus ${ }_{M}$ (Def. 4) can be employed.

One of the most used methods for evaluating segmentation is the figure of merit (fom) ([1,39]) defined in Def. 24. Be aware that the exact definition does not involve a difference to 1 , but it has been introduced to become a dissimilarity. The $\alpha$ parameter value is fixed to 1 without influence on the robustness of the criterion.

Definition 24 (Figure of Merit).

$$
\operatorname{fom}_{M}(X)=1-\frac{1}{\max \{\#(M), \#(X)\}} \sum_{p \in X} \frac{1}{1+\alpha \cdot d^{2}(p, M)}
$$

where $d(p, M)$ is the distance between the pixel $p \in X$ to the closest pixel of $M$ and \# the number of pixels of the considered mosaic $M$ or $X$ (which are not equal to $\mathcal{N}$ ).

A formulation that also uses the distance of a pixel $p$ to the closest one belonging to the reference $M$ can be found in [48]: 
Definition 25 (Yasnoff distance).

$$
\operatorname{yas}_{M}(X)=\frac{100}{\mathcal{N}} \sqrt{\sum_{p \in X} d^{2}(p, M)}
$$

Denoting $N_{o}$ is the number of over-segmented pixels $\left(N_{o}=\#(O)\right.$, with $\left.O=X \backslash M\right)$ and $N_{u}$ the number of under-segmented pixels $\left(N_{u}=\#(U)\right.$, with $\left.U=M \backslash X\right)$, [6] proposes two measurement methods as follows:

Definition 26 (Belaroussi criteria).

$$
\begin{aligned}
& o d i_{M}(X)=\frac{1}{N_{o}} \sum_{p \in O}^{N_{o}}\left(\frac{d(p, M)}{d_{T H}}\right)^{n} \\
& u d i_{M}(X)=\frac{1}{N_{u}} \sum_{p \in U}^{N_{u}}\left(\frac{d(p, X)}{d_{T H}}\right)^{n}
\end{aligned}
$$

The parameters $d_{T H}$ and $n$ establish a distance tolerance around the pixels of $M$. In the following, the values choosen are $n=4$ and $d_{T H}=\rho=2$ to be consistent with the $\epsilon$ dissimilarity criterion.

A more recent measure, denoted $j c d$ ([11], in reference to Jaccard, see also Def. 29) also uses the Euclidean distance to establish a criterion whose values are between 0 and 1 . Like fom (Def. 24), it has been modified to get a dissimilarity (Def. 27).

Definition 27 (Cárdenes measure).

$$
j c d_{M}(X)=1-
$$

$$
\frac{\#(M \cap X)}{\#(M \cap X)+\sum_{p \in X}\left(d^{2}(p, M)\right)+\sum_{p \in M}\left(d^{2}(p, X)\right)}
$$

12.4 Symmetric difference and derivatives

The following metrics are based on the symmetric difference (Def. 5).

The Nikodym metric is the measure of the symmetric difference ([31]), denoted niko $_{M}(X)$ :

Definition 28 (Discrete Nikodým metric).

$$
\operatorname{niko}_{M}(X)=\#(\Delta(M, X))
$$

The discrete Jaccard index is defined by Def. 29:

Definition 29 (Discrete Jaccard index).

$$
\operatorname{jac}_{M}(X)=\frac{\#(\Delta(M, X))}{\#(M \bigcup X)}=1-\frac{\#(M \bigcap X)}{\#(M \bigcup X)}
$$

Some very similar criteria have been proposed by [25] or referenced by [10] as overdetection or underdetection errors, and also as localization error ErrLoc (Def. 30):

Definition 30 (Localization error).

$$
\operatorname{ErrLoc}_{M}(X)=\frac{\#(\Delta(M, X)}{\mathcal{N}}
$$

In the case of mosaics (binary sets), the classical $L q$ distance is defined by:

Definition 31 ( $L_{q}$ metric).

$$
\begin{gathered}
L_{M}(X)=\left(\frac{\sum_{p}\left|I_{M}(p)-I_{X}(p)\right|^{q}}{\mathcal{N}}\right)^{\frac{1}{q}} \\
=\left(\frac{\#(\Delta(M, X))}{\mathcal{N}}\right)^{\frac{1}{q}}
\end{gathered}
$$

Notice that in the case of binary images, and $q=1$, the following relations holds: $L_{1 M}(X)=\operatorname{Err}_{\operatorname{Loc}}(X)$. 


\subsection{Pixel to pixel comparison}

A measure of quality has been proposed by [35], although its formulation will not be explained here because it would take too long in this article. It will be called mquality (it is called $R$ in the original article). This criterion includes neighborhood considerations.

Based on the Rènyi entropy $H_{\alpha},[4,26]$ propose some other measures that can handle grey level images (Küllback, Bhattacharyya and Jensen-like distances):

\section{Definition 32 (Küllback distance).}

$$
d k u_{M}(X)=\frac{1}{\mathcal{N}} \sum_{p}\left\{\left(I_{X}(p)-I_{M}(p)\right) \times \log \frac{I_{X}(p)}{I_{M}(p)}\right\}
$$

Definition 33 (Bhattacharyya).

$$
d b h_{M}(X)=-\log \left(\frac{1}{\mathcal{N}} \sum_{p} \sqrt{I_{X}(p) \times I_{M}(p)}\right)
$$

Definition 34 (Jensen-like distance).

$$
\operatorname{dje}_{M}(X)=J_{1}\left(\frac{I_{X}+I_{M}}{2}, I_{X}\right)
$$

with

$$
J_{1}\left(I_{M}, I_{X}\right)=H_{\alpha}\left(\sqrt{I_{X} \times I_{M}}\right)-\frac{H_{\alpha}\left(I_{X}\right)+H \alpha\left(I_{M}\right)}{2}
$$

and

$$
H_{\alpha}(I)=\frac{1}{1-\alpha} \log _{2}\left(\sum_{p} I(p)^{\alpha}\right)
$$

There might be some problems when using $d k u$ for zero valued pixels $p$. Practically, one can use $I_{X}(p)+1$ and $I_{M}(p)+1$. In the case of binary images (values are 0 or 1 ), the Küllback distance is equivalent to ErrLoc (see Prop. 14), this is why this criterion will not appear in the results.

\section{Proposition 14}

$$
d k u_{M}(X)=\frac{\log (2)}{\mathcal{N}} \#(\Delta(M, X))
$$

\section{Criteria normalization and calibration}

The main problem, when comparing different geometric dissimilarity criteria, is that the numerical values cannot be directly compared from one method to the other. First, it depends on the application field, the data acquisition type, the scale of observation and on the resolution. Second, it depends on the criterion itself.

Usually, researchers employ a normalisation method: the dissimilarity criteria are divided by a normalisation factor, that can be the number of pixels in the domain $S$ or the maximum value of the dissimilarity criterion. The key question raises: is it enough to compare dissimilarity criteria together ? The answer is negative. Dissimilarity criteria do not represent the same things and do not follow the same mathematical rules. This is why in this study, the dissimilarity criteria are calibrated.

Definition 35 (Normalized and calibrated criterion $\hat{c}$ ). $\hat{c}$ is the calibrated criterion $c$ normalised by $\omega_{c}$.

$$
\hat{c}=\frac{c}{\omega_{c}}
$$


The value 1 represents the criterion value above which two compared mosaics are different. 1 is thus a threshold value under which each criterion can be considered as valid. The calibration value $\omega_{c}$ will be defined in Def. 36.

For the choosen application domain, the human corneal endothelium, and for a given spatial resolution and scale factor, the criteria are calibrated with the following method: every mosaic from the database (Figs. 25 and 26) is compared to the 29 others. If $(i, j) \in[1 ; 30]^{2}\left(M_{i}\right.$ is a mosaic from the database), then the calibration value for a dissimilarity criterion $c$ is defined as the minimum of all comparisons for this criterion.

Definition 36 (Calibration value for a criterion). The calibration value, denoted $\omega_{c}$, for a given dissimilarity criterion $c$, is defined by:

$$
\omega_{c}=\min _{(i, j) \in[1 ; 30]^{2}}\left(c_{M_{i}}\left(M_{j}\right)\right)
$$

If the mosaics from the database are supposed to be different (which is realistic, see Fig. 25), the calibration value $\omega_{c}$ stands for the threshold value above which two mosaics should be considered different (see Fig. 10). The mean or the maximum value of all comparisons could have been used instead. But normalizing by the minimum value make the results more restrictive. The results for each criterion are presented in Table 1 .

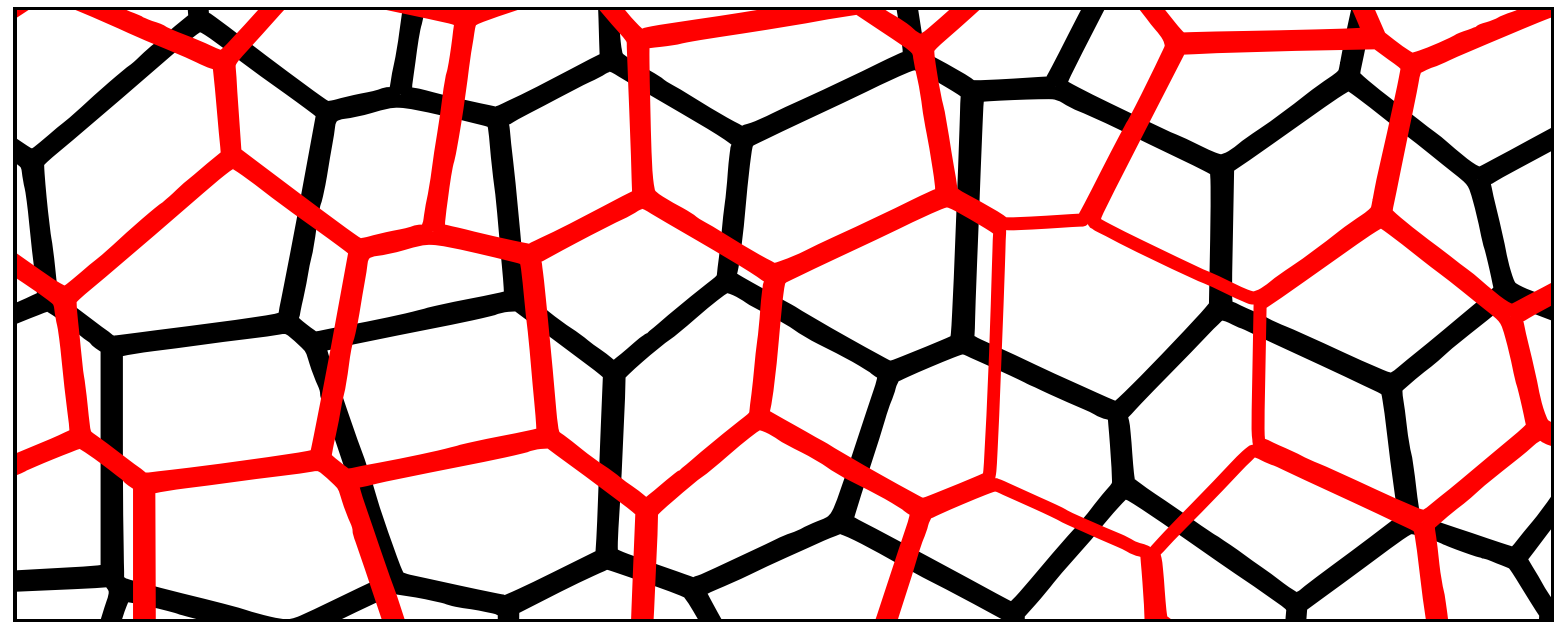

Fig. 10 Comparison of two different mosaics. Visually, these mosaics appear to be really different. Practically, let's say that a criterion $c$ applied on these mosaics gives a value of $\omega_{c}$. This means that if two different mosaics compared with the same criterion $c$ obtain a value above $\omega_{c}$, they can be considered as different.

\begin{tabular}{|c||c|}
\hline$c$ & $\omega_{c}$ \\
\hline \hline ErrLoc & 0.07 \\
\hline dbh & 2.06 \\
\hline Haus & 13.42 \\
\hline fom & 0.73 \\
\hline odi & 0.71 \\
\hline udi & 0.71 \\
\hline dje & 0.13 \\
\hline$\epsilon$ & 0.22 \\
\hline jcd & 1 \\
\hline yas & 0.0013 \\
\hline mquality & 5066 \\
\hline
\end{tabular}

Table 1 Table of the minimal values of the dissimilarity criteria $c$ when all couples of mosaics of the database are compared. The calibration value $\omega_{c}$ reflects the threshold value above which two compared mosaics should be considered as different. 


\section{Quantitative comparison of the 11 criteria}

With the 30 mosaics from the database, the dissimilarity criteria are numerically evaluated versus a degradation of the reference mosaic $M$. The operator $\mathcal{T}: X=\mathcal{T}(M)$ denotes the transformation (degradation) of $M$. This operator will act for over-segmentation, under-segmentation and small displacements (translation, distortion and smoothing).

\subsection{Under-segmentation and over-segmentation}

The under-segmentation operation consists in randomly choosing a pixel present in the reference mosaic $M$ (i.e. in the contours) and erasing it. Then, the considered contour is suppressed by using a morphological operation that performs a pruning. The result is equivalent to a deletion of one edge of a cell (Fig. 11).

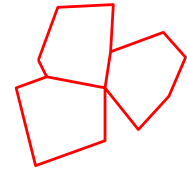

1. Reference mosaic

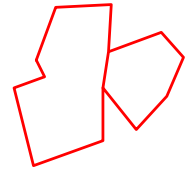

2. Undersegmentation of the reference mosaic

Fig. 11 Illustration of the under-segmentation process.

The over-segmentation operation consists in randomly picking up a cell in the mosaic, randomly choosing two pixels in it and then split the cell like a watershed operation would do (Fig. 12, see also [7]).

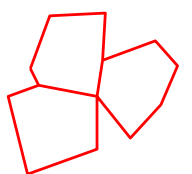

1. Reference mosaic

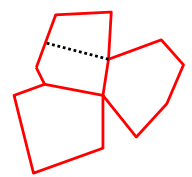

$2 . \quad$ Oversegmentation of the reference mosaic

Fig. 12 Illustration of the over-segmentation process.

Thus the function $\hat{c}$ is defined from the criterion $c$ as a function of its degradation $x$ :

Definition 37 (Mosaic degradation).

$$
x=\frac{\#(\mathcal{T}(M))-\#(M)}{\#(M)}
$$

where $M$ is the reference mosaic and $\tau(M)$ is the degraded mosaic, and \# means the number of pixels in a mosaic

Notice that $x$ is negative when $\mathcal{T}$ is an under-segmentation and positive for an over-segmentation.

Moreover, two quality measurements $q_{c, o}$ (over-segmentation quality) and $q_{c, u}$ (under-segmentation quality) are defined as follows:

Definition 38 (Quality measurements).

$$
\begin{aligned}
& q_{c, o}=100 \times \underset{x}{\arg \min }\{\hat{c}(x) \geq 1 \text { and } x \geq 0\} \\
& q_{c, u}=100 \times \underset{x}{\arg \max }\{\hat{c}(x) \geq 1 \text { and } x \leq 0\}
\end{aligned}
$$


Be aware that these quality measurements are defined for one particular mosaic and may slightly vary from one mosaic to the other. The mean results for the 30 mosaics of the database are presented in Table 2.

\begin{tabular}{|c||c|c|}
\hline \multicolumn{1}{|c||}{} & $\hat{q}_{o}$ & $\hat{q}_{u}$ \\
\hline \hline ErrLoc & & 83 \\
\hline dbh & $N A$ & 80 \\
\hline Haus & -1 & 0.6 \\
\hline fom & & 74 \\
\hline odi & -1 & $N A$ \\
\hline udi & $N A$ & 0 \\
\hline dje & & 80 \\
\hline$\epsilon$ & & 100 \\
\hline jcd & & 100 \\
\hline yas & -34 & $N A$ \\
\hline mquality & & 53 \\
\hline
\end{tabular}

Table $2 \hat{q}_{u}$ and $\hat{q}_{o}$ are the mean quality measurements defined for under or over segmentation (see Sect. 14.1) of the 30 mosaics of the database. This cannot prove that a criterion is good, but it can show that a criterion behaves poorly (as Haus, odi, udi and yas).

A cell with no value means that it could not be computed (there was no computed value $x$ that could define $q_{u}$, but it may exists). In particular, some cells show $N A$, which means that a specific criterion cannot measure under or over-segmentation.

The results are presented in Figs. 13, 14 and 15. $x$ is represented in absciss, and $\hat{c}(x)$ in ordinate. In Fig. 13, all comparison criteria are represented for the degradation (under and over-segmentation) of only one mosaic. The clouds of points represented in Fig. 15 reflects the degradation of all mosaics from the database (only one time). The Fig. 14 shows the degradation for mosaic 1 of the database.

\subsection{Translation}

This operation is the translation $\tau$ of a mosaic in the four directions right, left, up and down of a rectangular grid (see Fig. 16). A small translation (of a few pixels) should give a small criterion value (i.e. less than $\omega_{c}$ ). The results are presented in Fig. 17: translations $\tau(n)$ of $n$ pixels are used. The mean value for the 4 directions is shown, normalized by $\omega_{c}$. Still, all values below 1 mean that the compared mosaics can be considered as similar (in other words cannot be considered as dissimilar).

The considered corneal mosaics are particular in the way they present a regular pattern (something like an hexagon, that represents a cell) that is reproduced in the image. This means that if a translation of about the size of a cell is performed, the translated mosaic may overlap with the original one. This explains the fact that the comparison values seem to tend to a specific value (Fig. 17).

\subsection{Distortion}

If a mosaic is approximated by polygonal lines ([36] and QGAR library ${ }^{1}$ ), the borders of the cells are the edges and their intersections are the vertices. What is called a distortion is a random displacement of each vertex in a square neighborhood of a given size (see Fig. 18).

Definition 39 (Distortion formulation). Let $\tilde{M}=\left(V_{M}, E_{M}\right)$ be the polygonal approximation of the reference mosaic $M . V_{M}$ is the set of vertices, and $E_{M}$ the set of edges. Each vertex $v$ of $V_{M}$ has two coordinates in $2 \mathrm{D}, v_{x}$ and $v_{y}$. The distortion $\mathcal{D}$ of size $s$ is defined as follows:

$$
V_{\mathcal{D}(M, s)}=\left\{v^{\prime}=\left(v_{x}+r_{v_{x}}, v_{y}+r_{v_{y}}\right)\right\}
$$

where $r_{v_{x}} \in \mathbb{N}$ and $r_{v_{y}} \in \mathbb{N}$ are random variables in $[-s ; s], s \in \mathbb{N}$.

\footnotetext{
1 http://www.qgar.org
} 


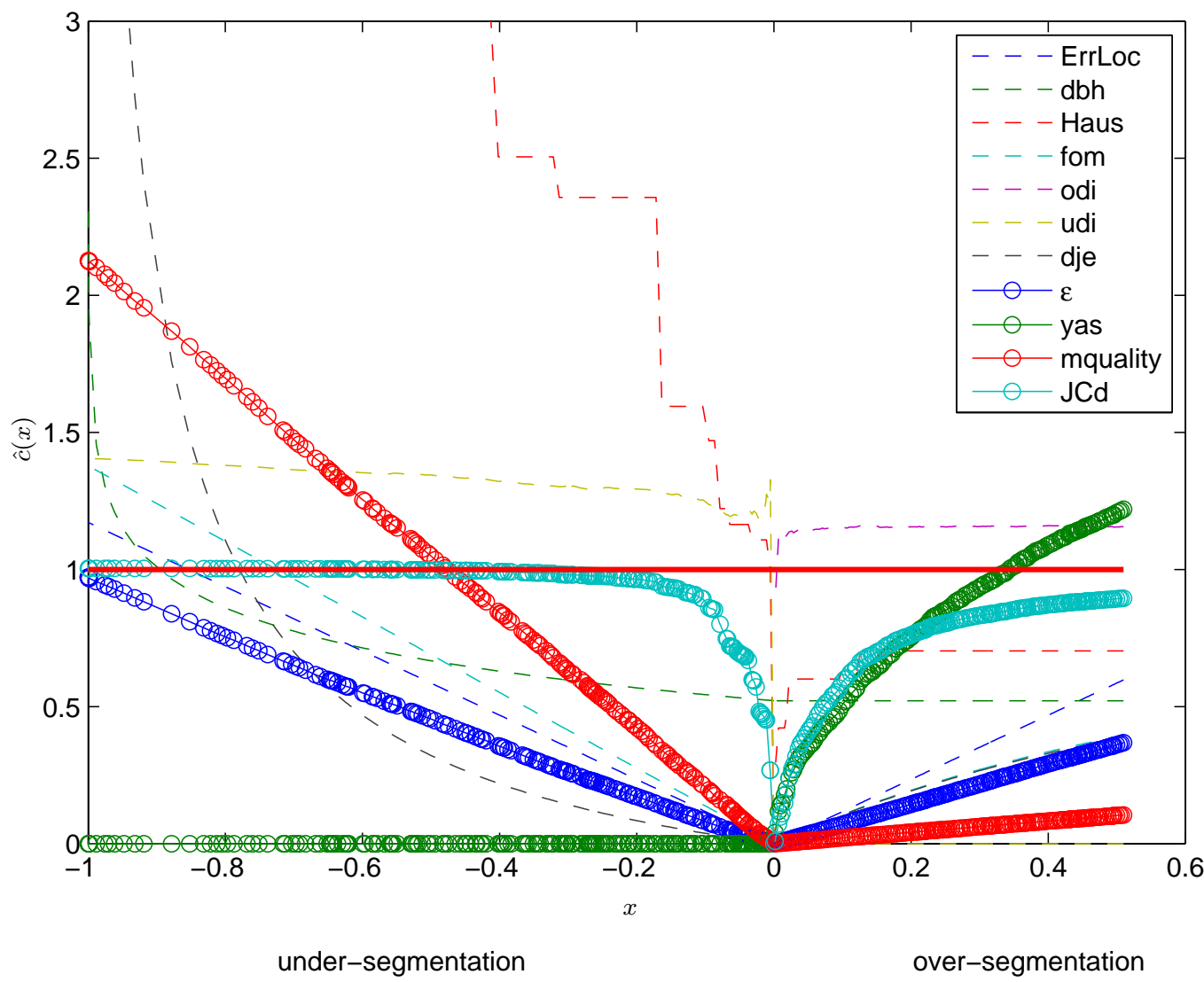

Fig. 13 Representation of $\hat{c}(x)$ for the 11 dissimilarity criteria. The values $q_{c, o}$ and $q_{c, u}$ can be observed when the curve associated to a criterion $c$ is crossing the horizontal line in red defined for the value 1 (when applicable). Increasing criteria that stay most of the time under the value $1\left(\hat{c} \leq 1 \Leftrightarrow c \leq \omega_{c}\right)$ are to be preferred. See Fig. 14 for detailed diagrams.

This can be seen as the errors made by an expert when manually drawing the contours. The results of 3 experiments are presented in Figs. 19,20 and 21, respectively on a mosaic with small, median and big cells. After this distortion, the mosaic $\tilde{M}$ is transformed into a discrete mosaic by drawing the edges of $E_{\mathcal{D}(M, s)}$ as lines (this defines another discrete mosaic).

The criterions that can be used to evaluate the distortion of the mosaics have to be increasing for the parameter $s$ and stay a long time under the value 1. For example, ErrLoc, mquality and dje present for $s>=1$ some values greater that 1 , which means that they are not adapted to this distortion evaluation. Be aware that this is not visible for ErrLoc and mquality in Fig. 21, because the value $s$ is lower relatively to the big cell size than in Figs. 19 and 20.

\subsection{Smoothing}

The smoothing process $\mathcal{S}$ consists in getting a smooth mosaic from the original one. There would be a lot of different ways of getting a so-called smooth mosaic, for example using deformable models. We choosed to use some mathematical morphology operations. The mosaic $M$ is first dilated (see the Minkowski addition Def. 8) with a ball structuring element $B$ of size $s(B=s N)$. Then, the result is thinned and the spurs are removed. The obtained mosaic is a smooth version approximating the original one. The Figs. 22, 23 and 24 illustrate this transformation for cells of small, median and big sizes. What can be observed in these graphs is that for some value $s$ of the dilation, the $\hat{c}$ is near or above 1, which means that the mosaics are really damaged. The second interesting criterion is the monotonicity: some criterions have not increasing values and thus are not usefull for this type of transformation. 


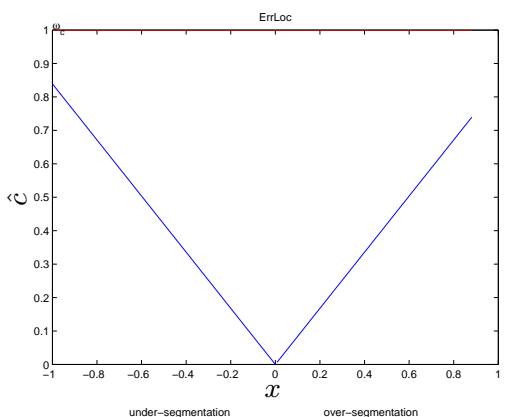

under-segmentation $x$ over-segmentation

1. ErrLoc: $\operatorname{good} q_{c, u}$ and $q_{c, o}$

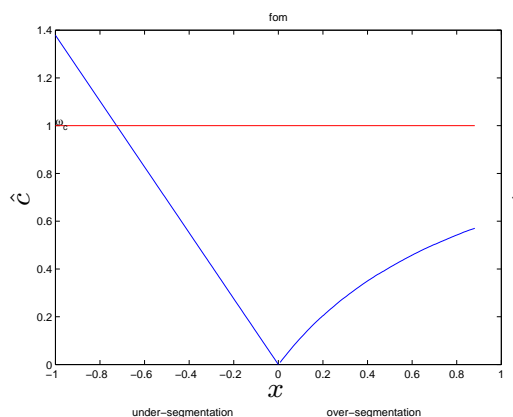

fom: good $q_{c, u}$ and $q_{c, o}$

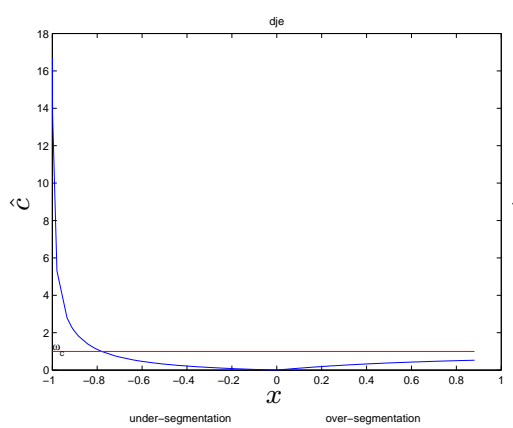

7. dje: $\operatorname{good} q_{c, u}$ and $q_{c, o}$

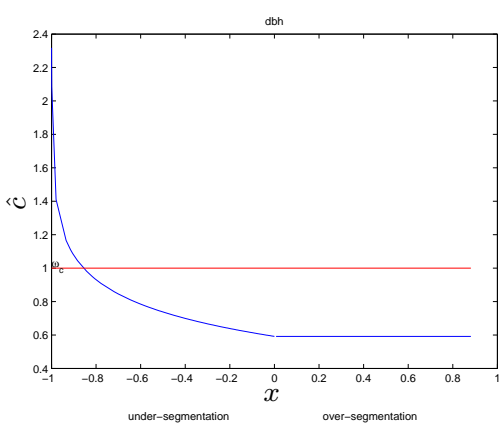

2. $d b h: \operatorname{good} q_{c, u}$, poor $q_{c, o}$

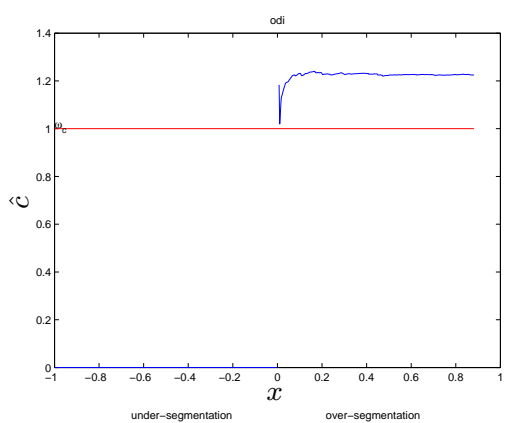

5. odi: poor $q_{c, u}$ and $q_{c, o}$

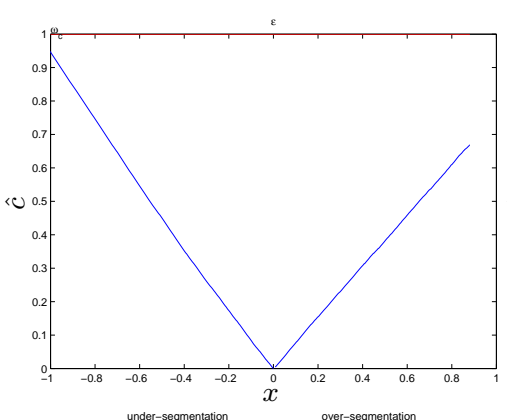

8. $\epsilon: \operatorname{good} q_{c, u}$ and $q_{c, o}$

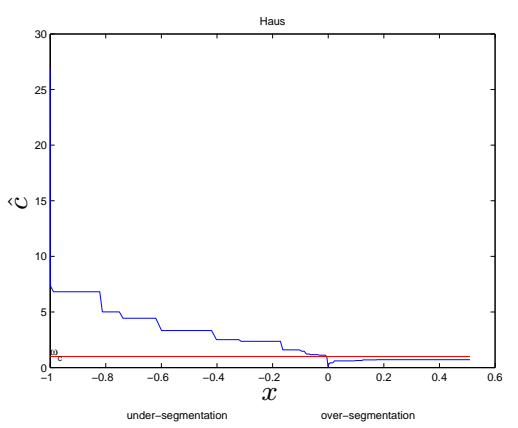

3. Haus: poor $q_{c, u}$ and $q_{c, o}$

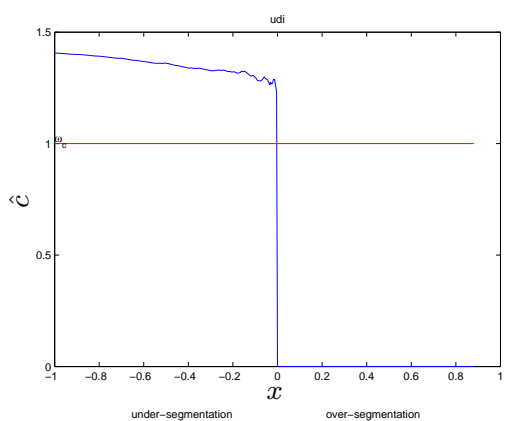

6. udi: poor $q_{c, u}$ and $q_{c, o}$

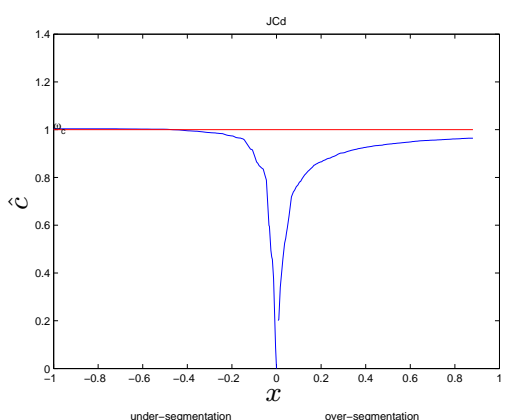

9. jcd: good $q_{c, u}$ and $q_{c, o}$, (although very steep)

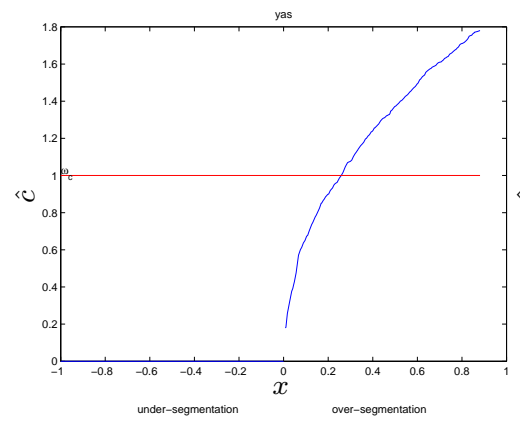

10. yas: poor $q_{c, u}$, good $q_{c, o}$

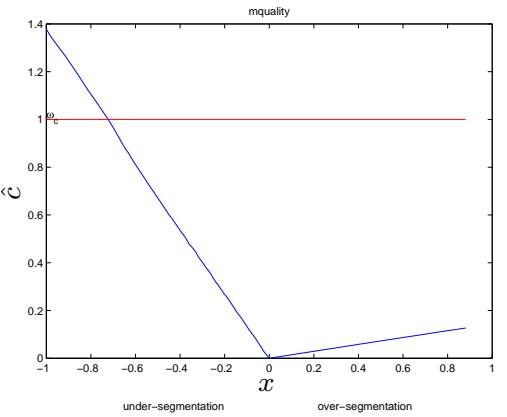

11. mquality: good $q_{c, u}$ and $q_{c, o}$ (although very flat)

Fig. 14 Representation of $\hat{c}(x)$ for different criteria $c$ and for mosaic 1 of the database (Fig. 25). Especially for undersegmentation, the criterion $c$ can be trusted when $\hat{c}(x) \leq 1$. The quality measurements $q_{c, u}$ and $q_{c, o}$ are computed with these data. 

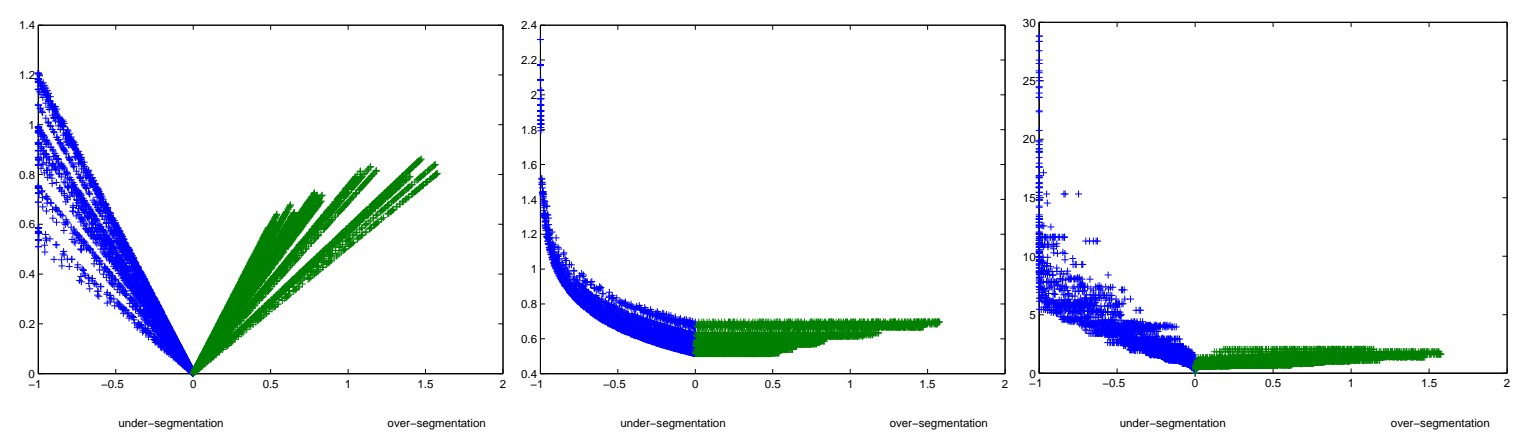

1. ErrLoc

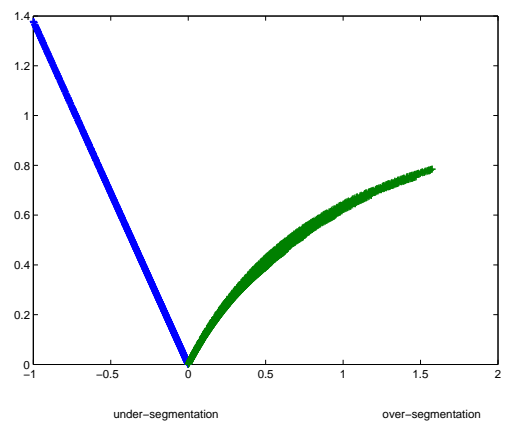

2. $d b h$

3. Haus

4. fom
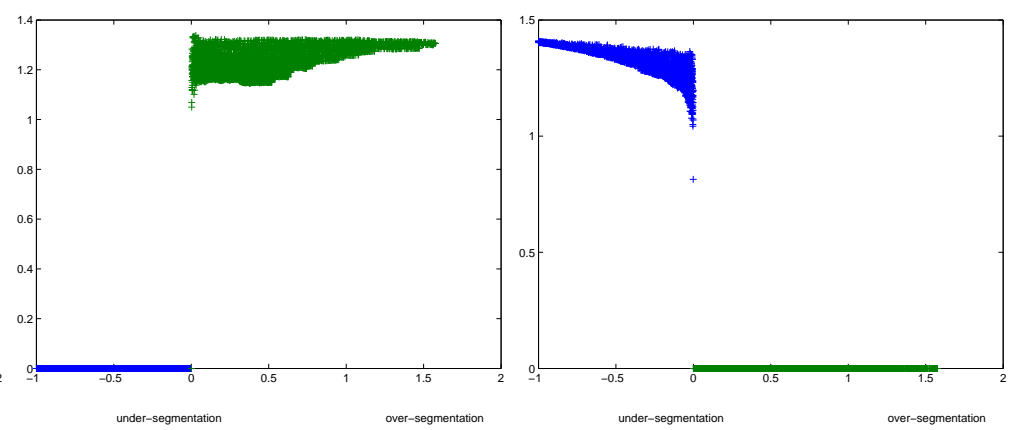

5. odi

6. $u d i$

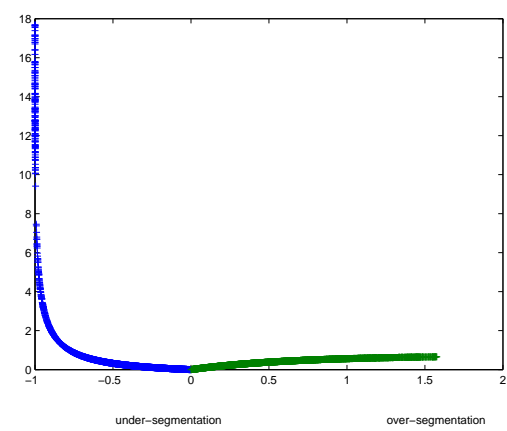

7. dje

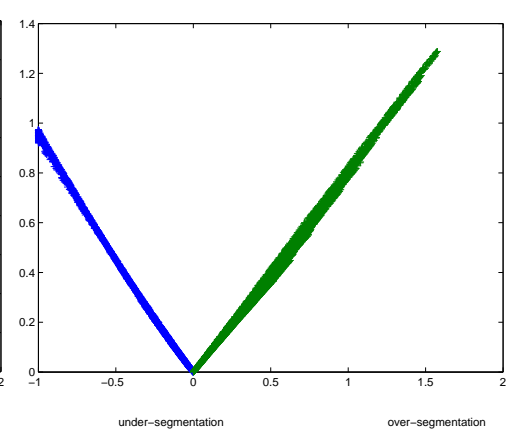

8. $\epsilon$

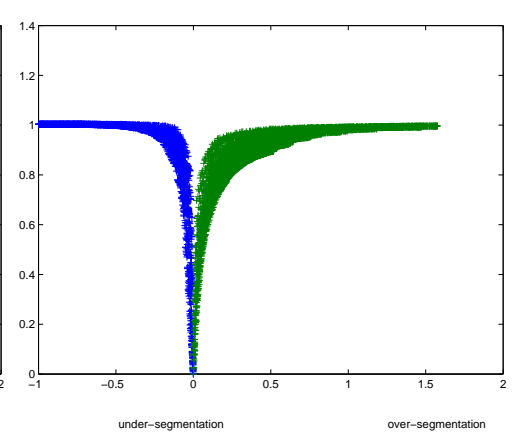

9. jcd

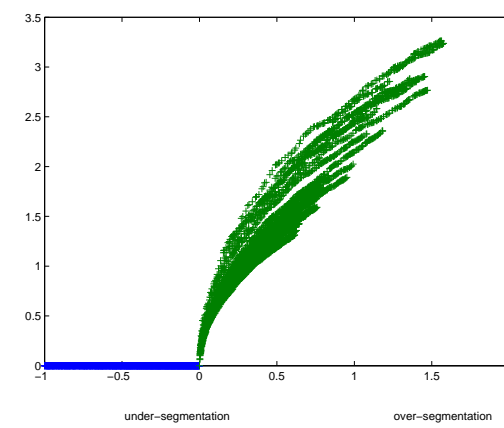

10. yas

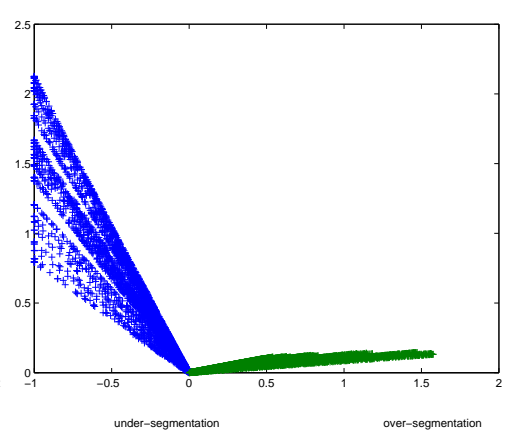

11. mquality

Fig. 15 Representation of $\hat{c}(x)$ for different criteria $c$ and for the 30 images of the mosaic database (Fig. 25). 


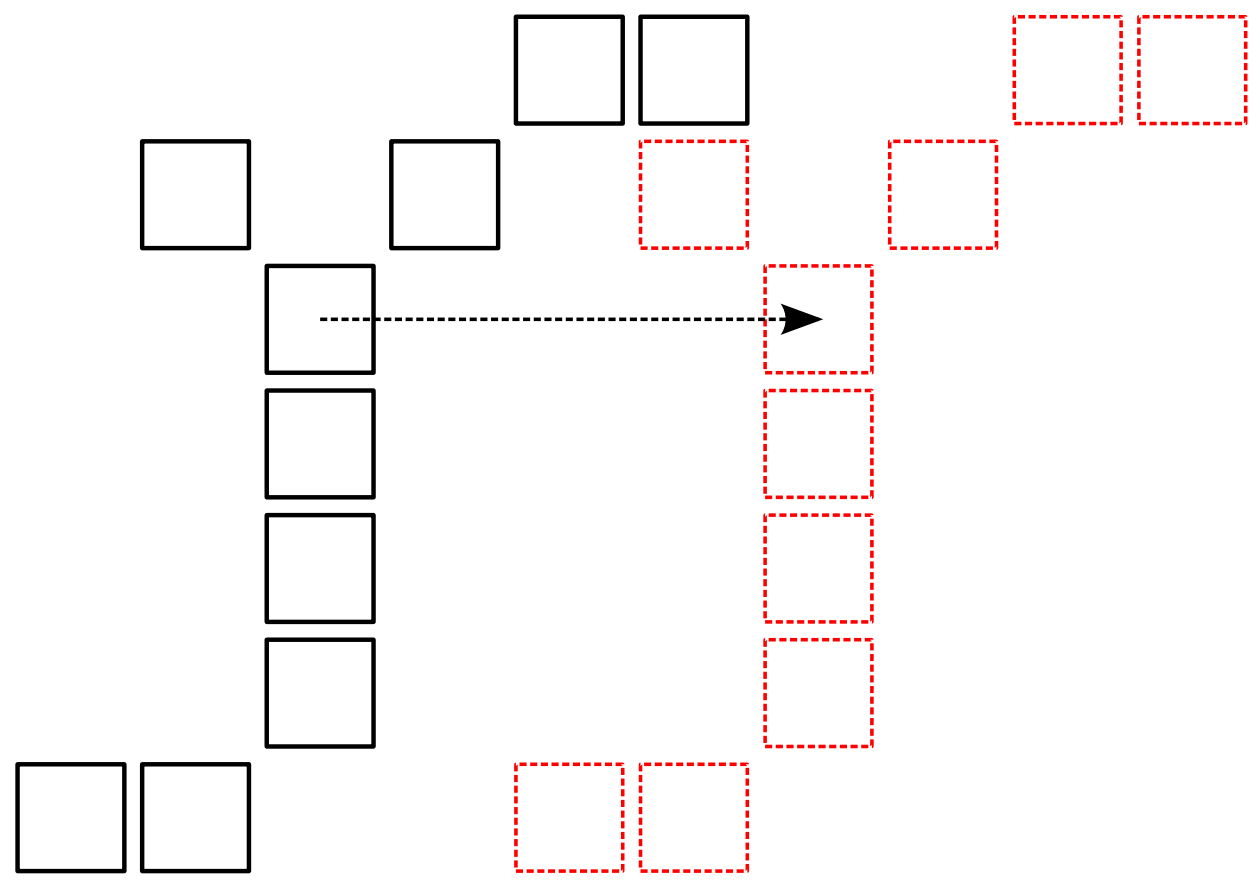

Fig. 16 Illustration of a translation on a piece of a mosaic. This is a rigid transformation of the mosaic.

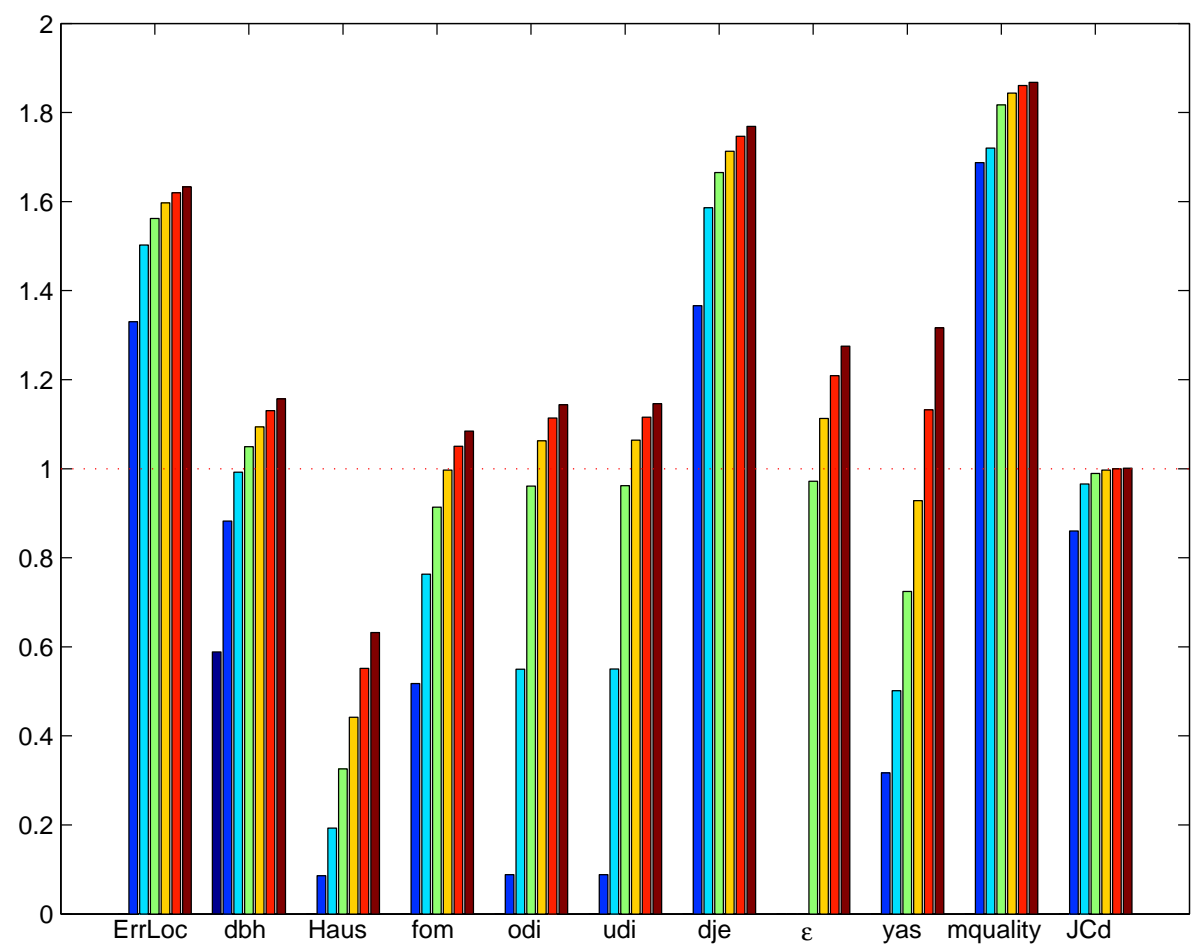

Fig. 17 Robustness of the 11 criteria versus translation. Each bar represents the mean criterion value in the four directions for the 30 mosaics of the database. For each criterion, translations from 1 to 6 pixels have been represented in colorbars.

To understand this graph, notice that as these mosaics represent a regular tiling of some sort of cells, after a translation of a cell size the translated and the original mosaic may be superposed. This means that after such a translation, both mosaics begin to be visually really dissimilar and a criterion should reflect it (and also present values above 1). This shows that the Hausdorff criterion cannot correctly evaluate translations because it is bounded in the case of mosaics. 


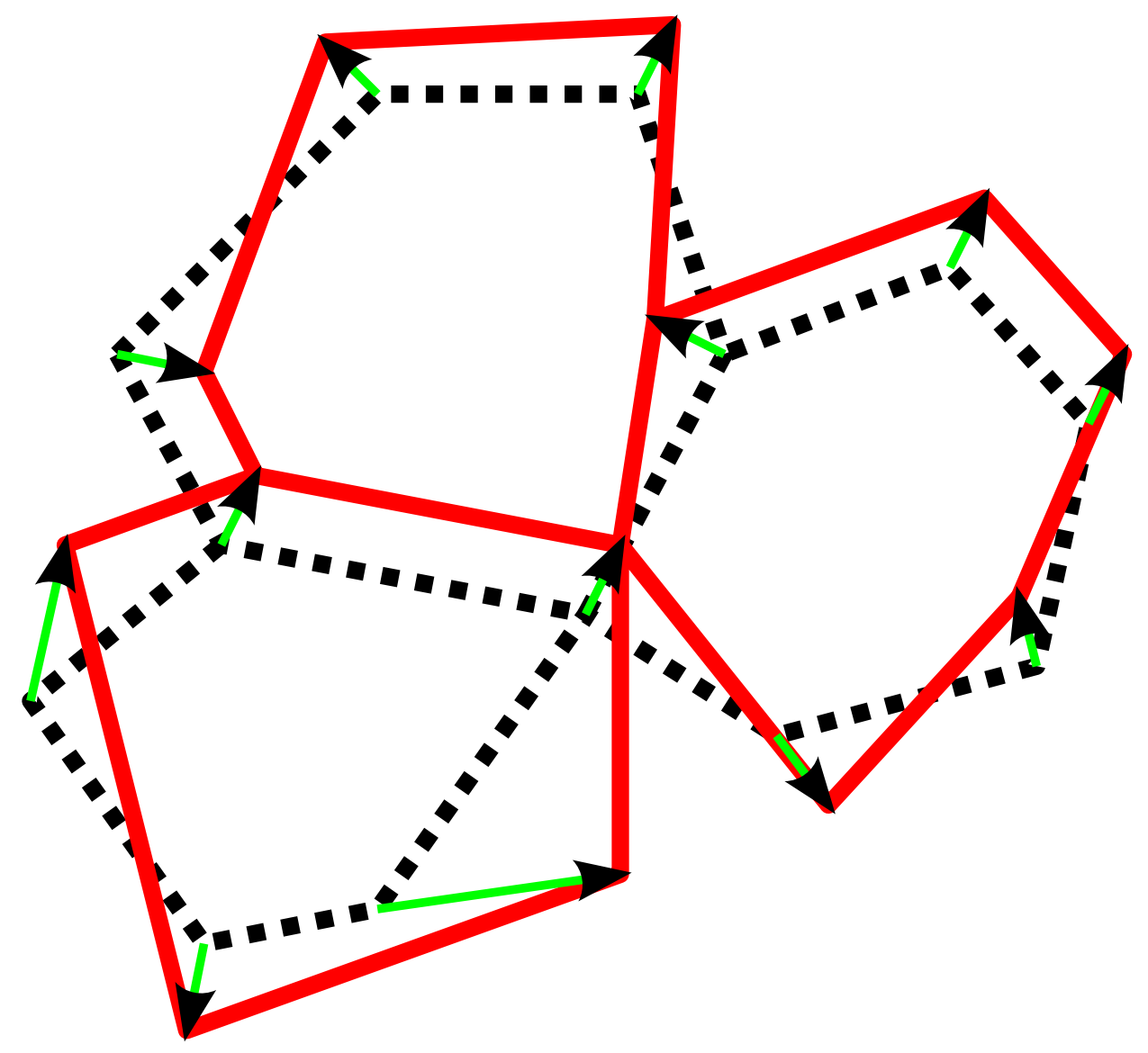

Fig. 18 Illustration of the distortion process. Each vertex of the mosaic is randomly moved in a given neighborhood. The comparison of the dotted mosaic and the continuous mosaic is then performed.

\subsection{Small spatial variations}

This section does not present a transformation that will allow the measurement of the tolerance of the different criteria to small variations. It emphasizes in the previous transformations where this behaviour can be observed.

A small spatial variation can be explained as the variation observed when an expert manually draws twice the same mosaic (with visually the same result). Blatantly, it depends of the scale of observation. It is related to the choice of the tolerance parameter $\rho$ for the $\epsilon$ dissimilarity criterion. For example in our application, a translation of less than $\rho$ pixels is considered as a small variation.

Thus, the tolerance to small variations for the different dissimilarity criteria can be observed on the graph of translation evaluation (Fig. 16) and on the graphs of distortion (Figs. 19,20 and 21). Only three criteria are tolerant (they are defined to be tolerant): udi, odi and $\epsilon$.

\section{Discussion and conclusion}

The aim of this second part of this article was to compare 11 dissimilarity criteria and to be able to choose the bests in the case of mosaics comparisons. In the cited research works that also perform dissimilarity criteria comparisons, the frustration came because the normalisation (and the comparison) was not really convincing. A normalisation between 0 and 1 is sometimes not possible, and generally not enough to allow a comparison. This is why a calibration method was introduced, that can highlight the values where we can be sure that the criterion values reflect different mosaics. 


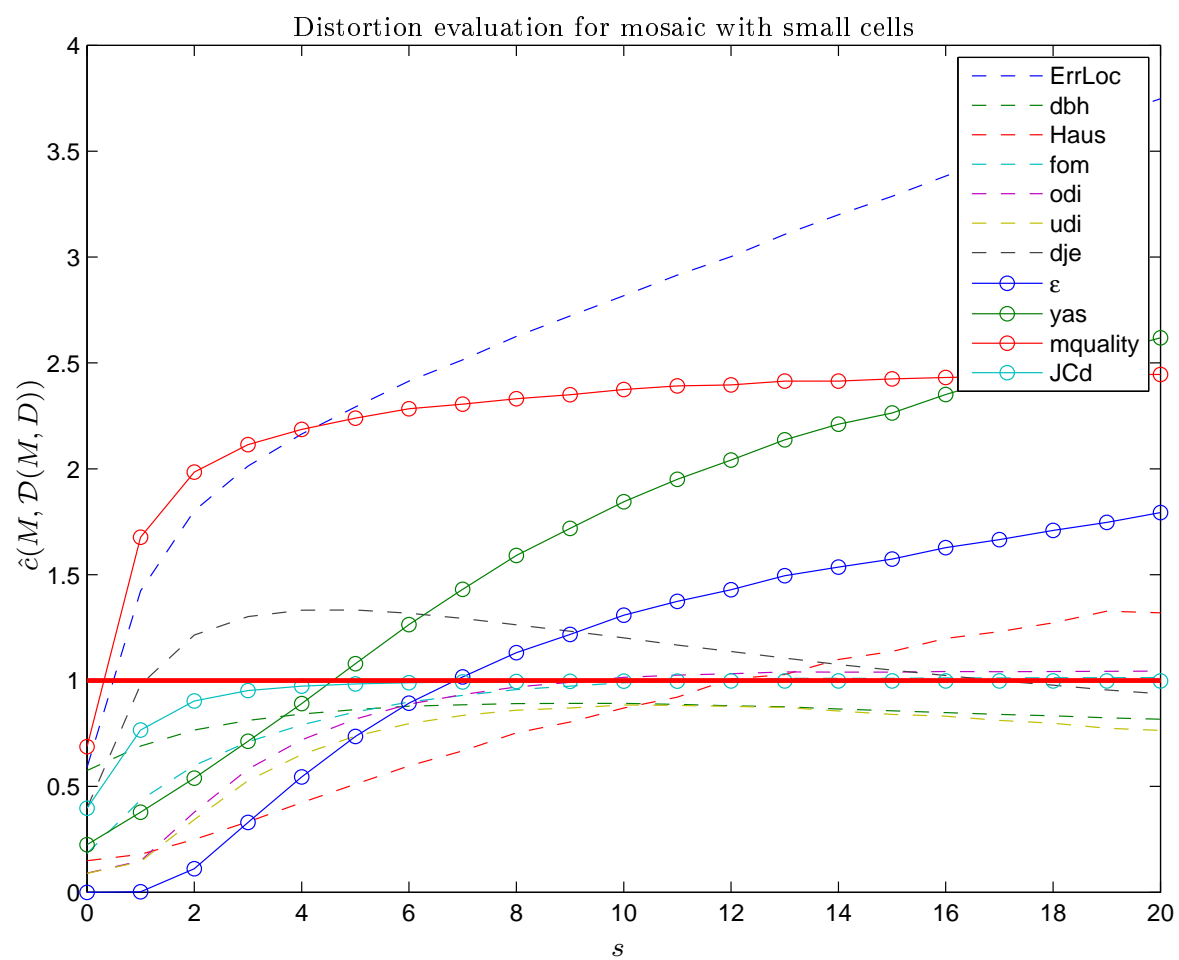

Fig. 19 Evolution of the criteria for one random distortion $\mathcal{D}(M, s)$ with the size $s$ represented in abscisse. This graph shows for $s=0$ that the lower measures are proposed by the criteria designed to be more tolerant ( $\epsilon$, udi, odi). Due to the polygonalization process, notice that the mosaic issued from the distortion $\mathcal{D}(M, 0)$ of size $s=0$ is not equivalent to the mosaic $M$. This explains the non null values for all the dissimilarity criteria but the $\epsilon$ dissimilarity criterion (that is designed to handle this approximation). The $\epsilon$ criterion also presents a null value for the translation of $s=1$ pixels as a result of the tolerance parameter ( 2 pixels of tolerance).

The comparison of the distortion for two different mosaics has no meaning because these mosaics present spatial structures (the cells) of different sizes. This is why this Fig. is only for mosaic 1 (with small cells). Figs. 21 and 20 present results for respectively big and median size cells.

The Table 3 summaries the different experiments presented to test the robustness of the criteria versus over and under segmentation, translation, distortion and tolerance to small variations. The presence of a green cell (with $\mathrm{a}^{*}$ ) indicates that the criterion is efficient to evaluate the considered transformation.

\section{Perspective}

A perspective already mentionned is to developp a method to automatically choose the tolerance parameter value, either by an observation of the mosaic or by some other informations (like the original image it might come from).

The $\epsilon$ geometric dissimilarity criterion was initially designed to tune up algorithms of corneal endothelium image segmentation ([17]). It is now planned to conduct a survey on segmentation methods of corneal images in order to compare their results together and propose adapted values for the operating parameters they could use. 


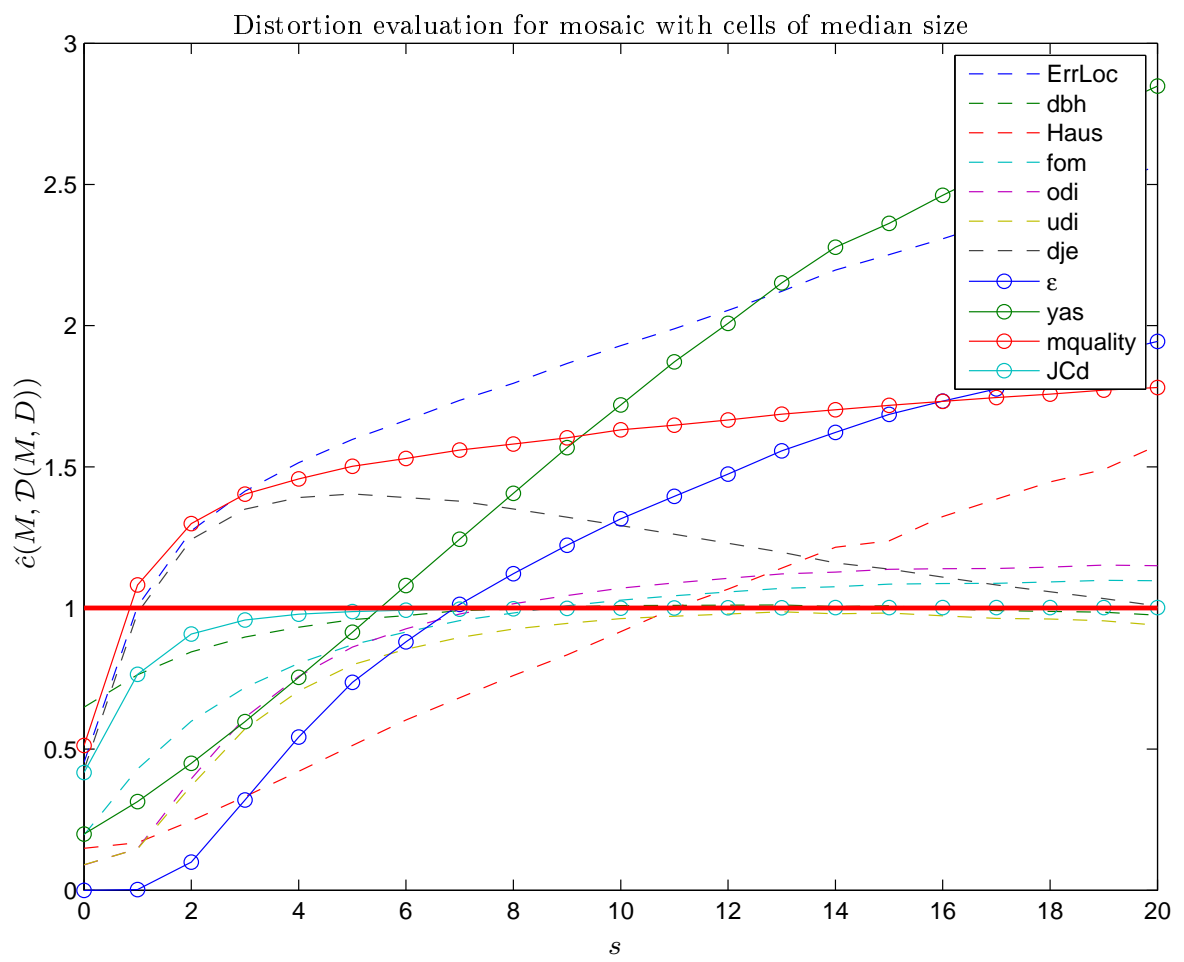

Fig. 20 Distortion evaluation for mosaic 26 (with cells of median size).

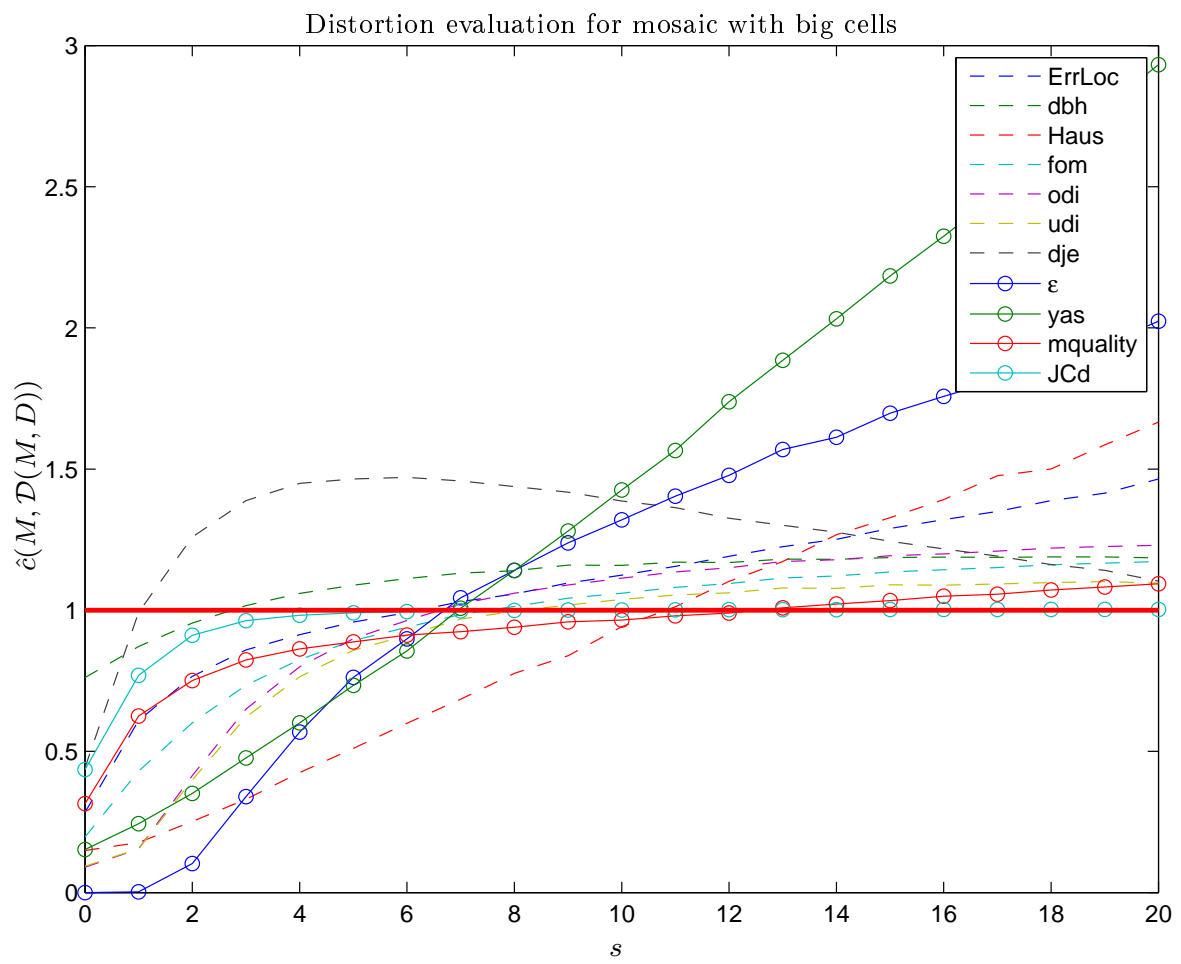

Fig. 21 Distortion evaluation for mosaic 11 (with cells of big size). 


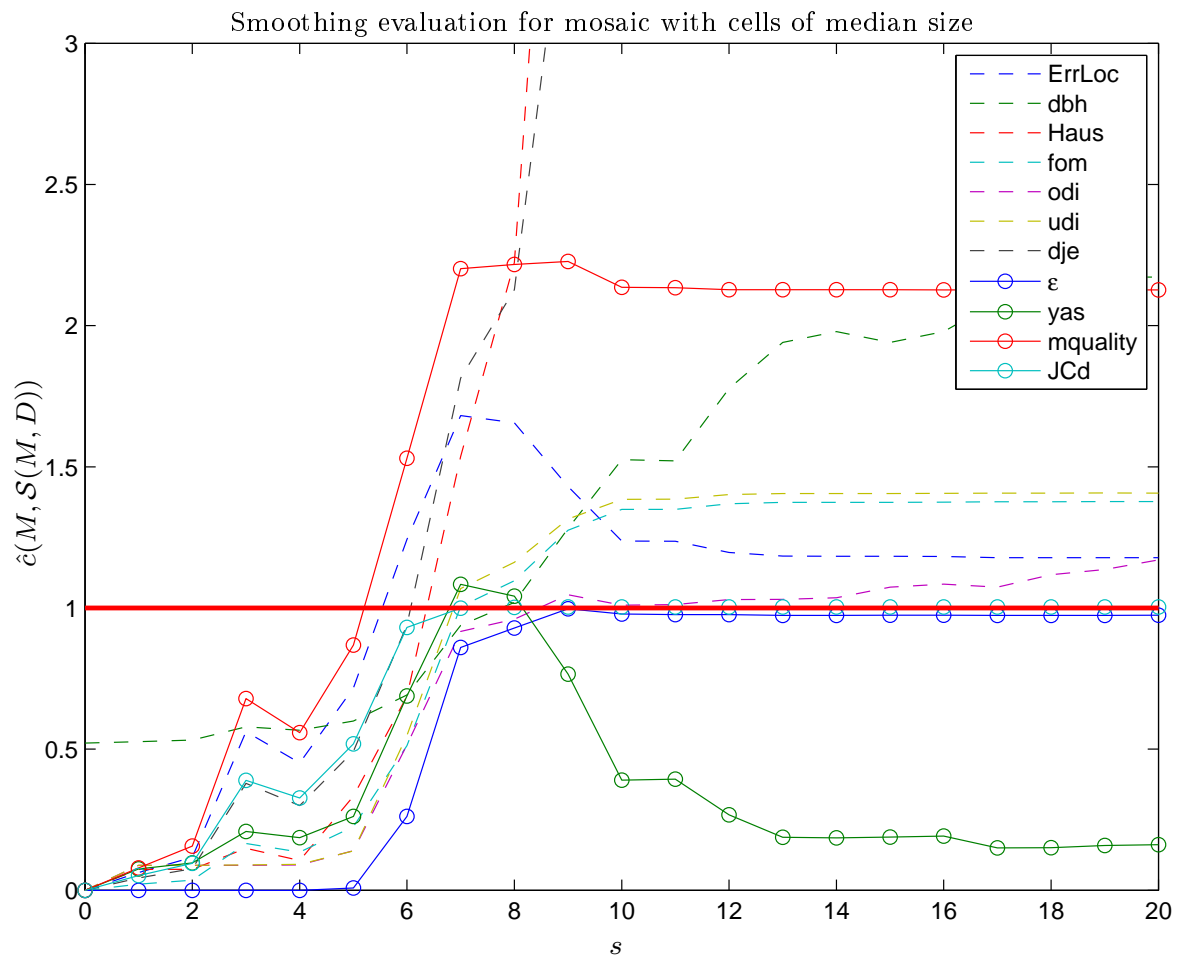

Fig. 22 Smoothing evaluation for mosaic 1 (with cells of small size). The parameter $s$ is the size of dilation used to smooth the mosaic.

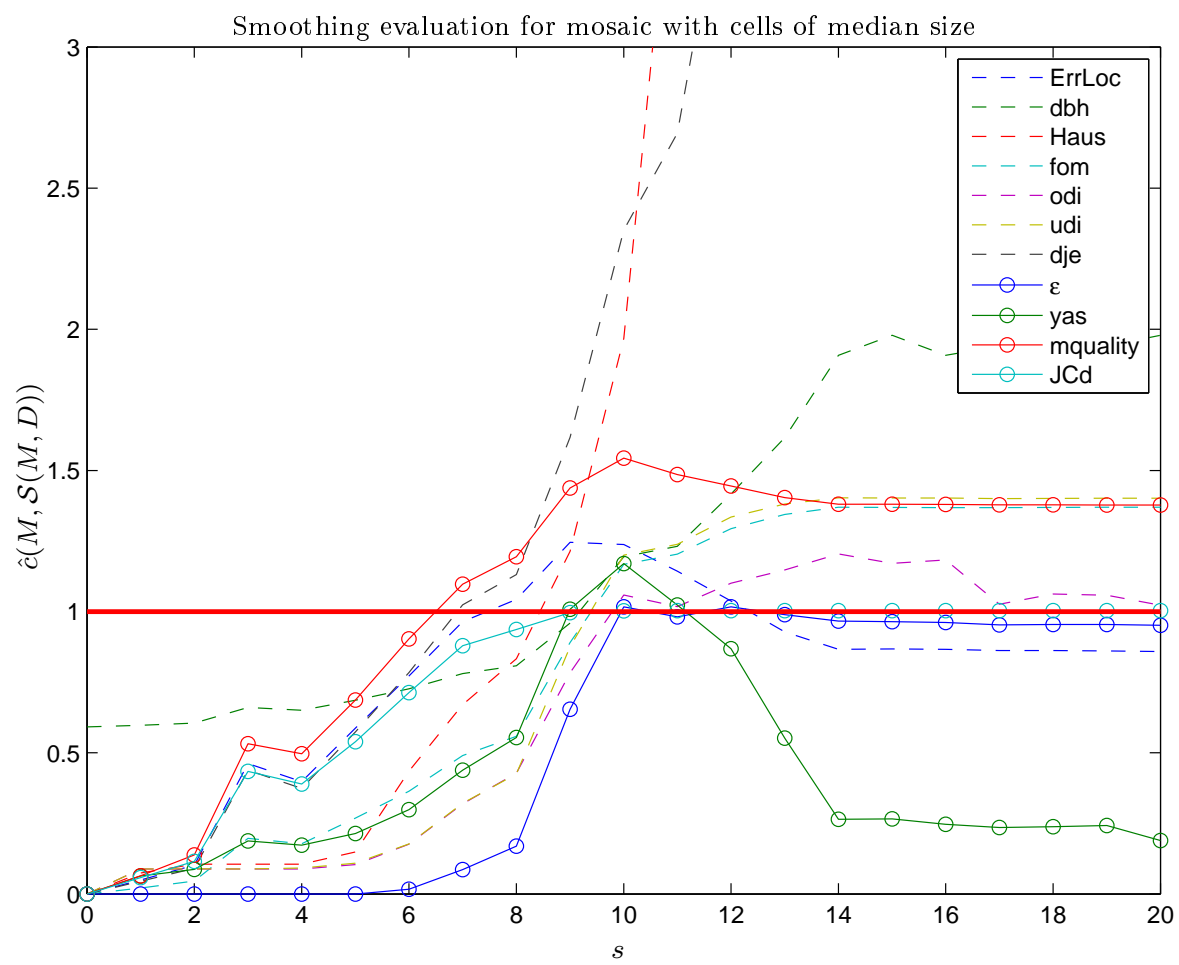

Fig. 23 Smoothing evaluation for mosaic 26 (with cells of median size). 


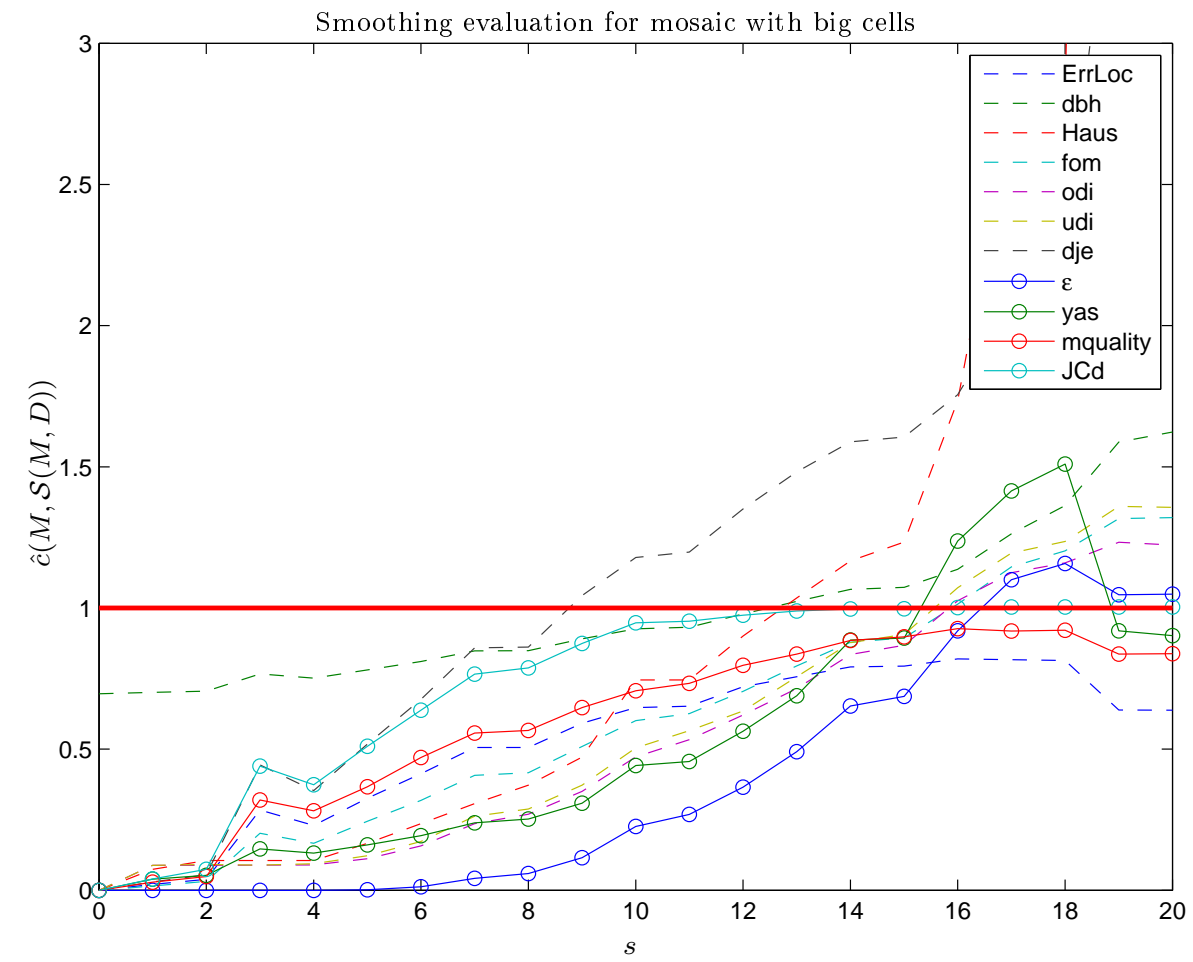

Fig. 24 Smoothing evaluation for mosaic 11 (with cells of big size).

\begin{tabular}{|c|c|c|c|c|c|c|}
\hline $\mathrm{c}$ & 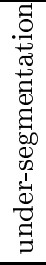 & 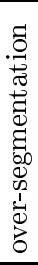 & 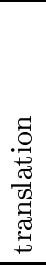 & 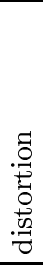 & 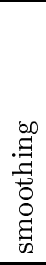 & 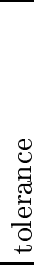 \\
\hline ErrLoc & * & * & & & & \\
\hline $\mathrm{dbh}$ & $*$ & & $*$ & $*$ & $*$ & \\
\hline Haus & & & $*$ & $*$ & & \\
\hline fom & * & * & $*$ & $*$ & & \\
\hline odi & & & $*$ & $*$ & $*$ & * \\
\hline udi & & & $*$ & $*$ & $*$ & * \\
\hline dje & * & $*$ & & & & \\
\hline$\epsilon$ & * & $*$ & * & $*$ & * & * \\
\hline jcd & * & $*$ & $*$ & $*$ & & \\
\hline yas & & * & * & $*$ & & \\
\hline mquality & * & * & & & & \\
\hline
\end{tabular}

Table 3 Summary table of the compared criteria and their observed robustness versus five transformations. A star (green cell) means that a dissimilarity criterion is able to discriminate the considered transformation. 


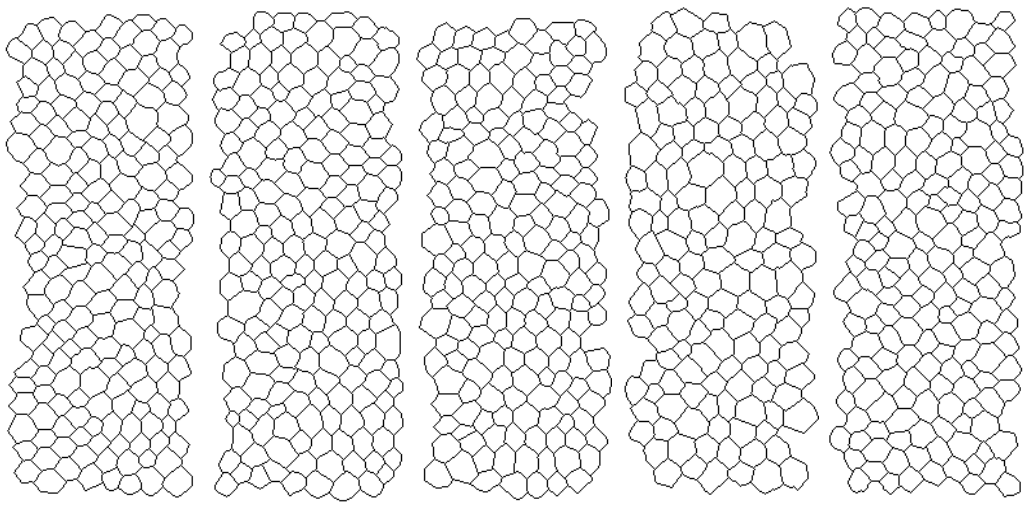

1.

3.

5.

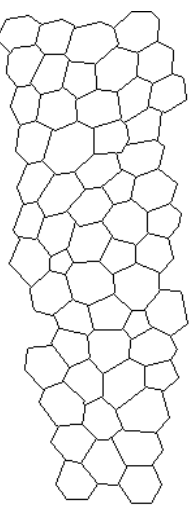

15.

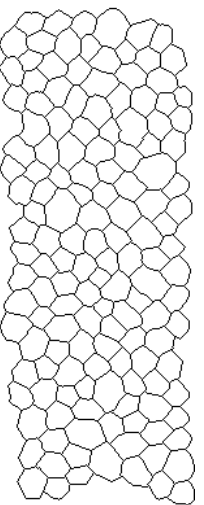

6.

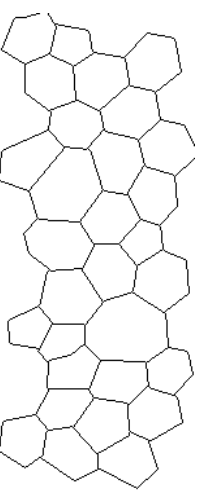

16.

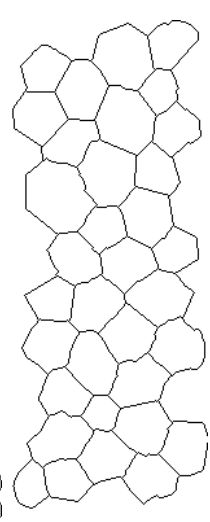

7.

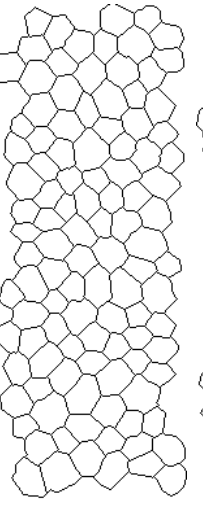

8.

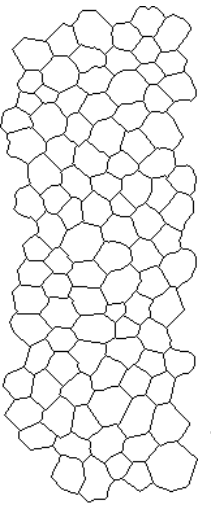

9.

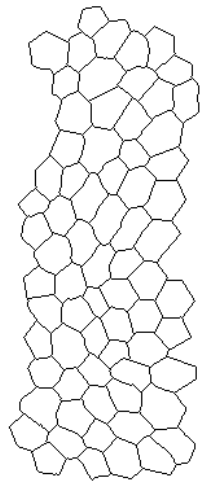

10.

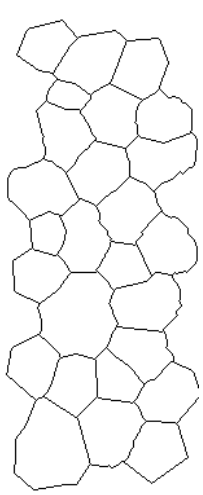

11.
12
13.

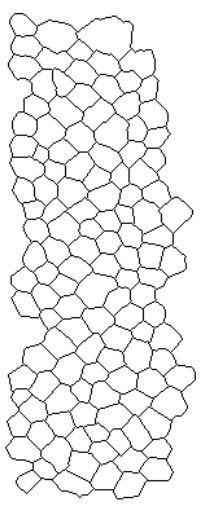

17.

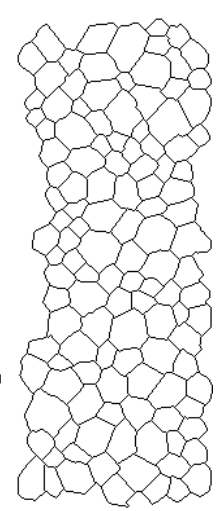

18

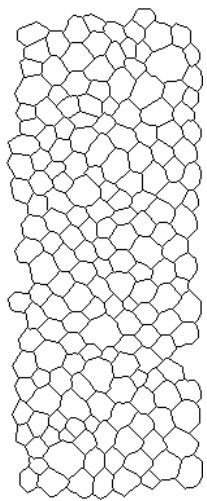

19.

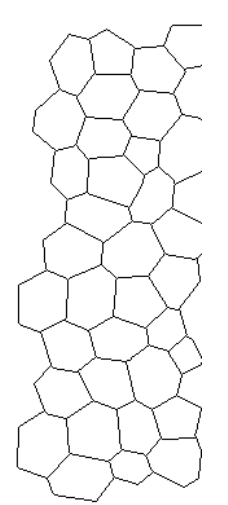

20.

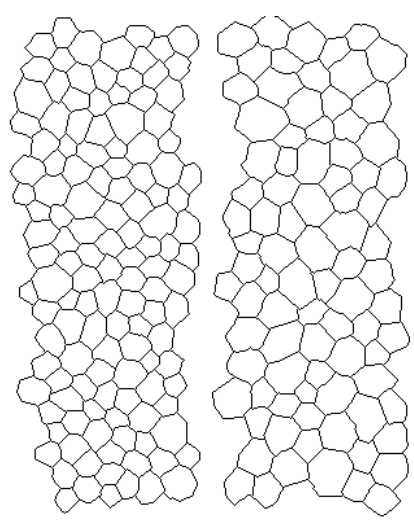

21.

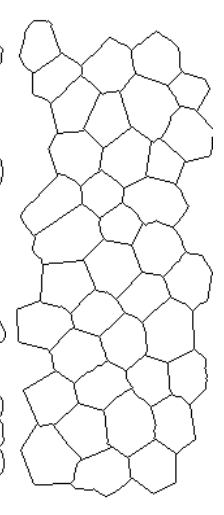

23.

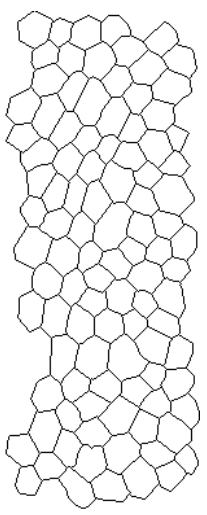

24.

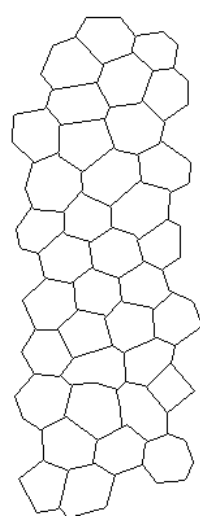

25.

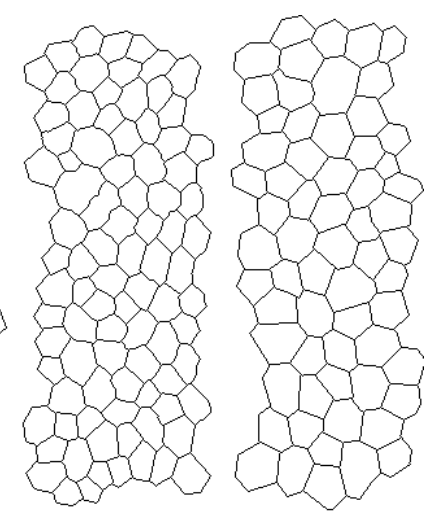

26.

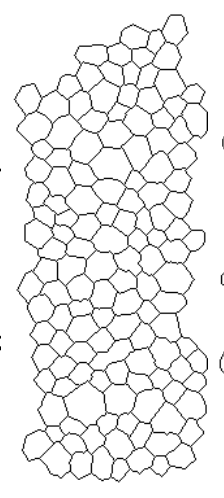

28.

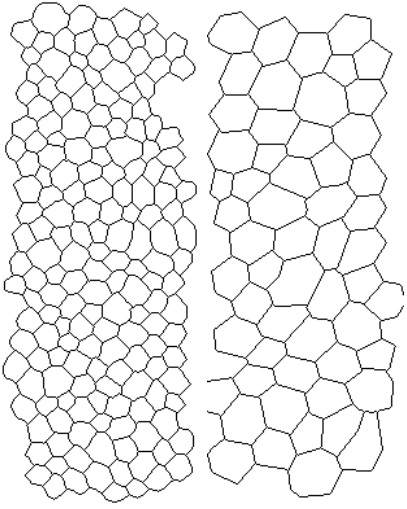

29.

Fig. 25 Table of the 30 mosaics of the database. They have been manually drawn by an expert ophthalmologist from a human corneal endothelium image database. The lines represent the contours of the cells. 

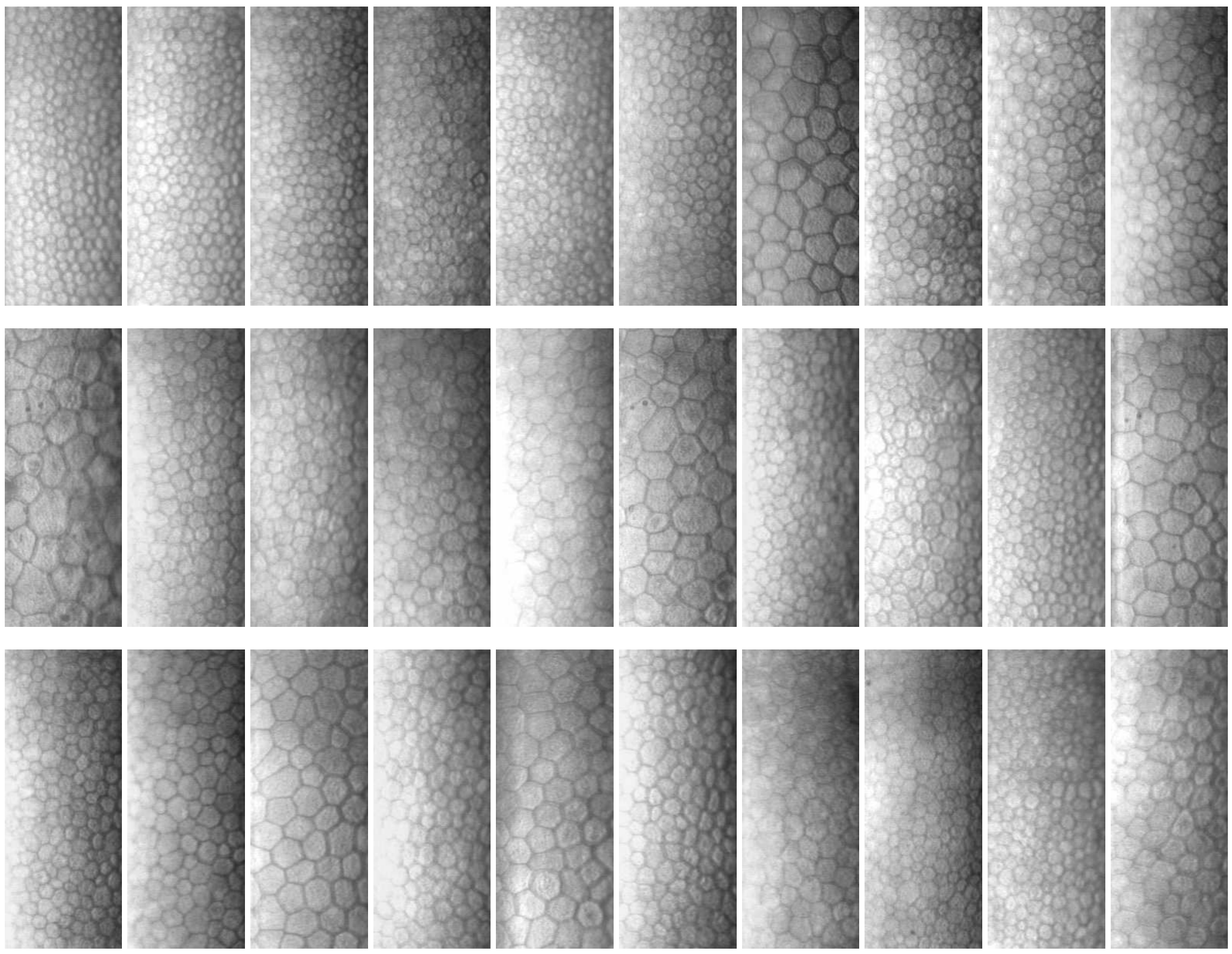

Fig. 26 Table of the 30 images of corneal endotheliums of the database, acquired in specular microscopy. 


\section{References}

1. Abdou, I., Pratt, W.: Qualitative design and evaluation of enhancement/thresholding edge detector. Proc. IEEE. 67(5), 753-763 (1979)

2. Ambrosio, L., Colesanti, A., Villa, E.: Outer minkowski content for some classes of closed sets. Mathematische Annalen 342(4), 727-748 (2008)

3. Baddeley, A.J.: An error metric for binary images. In: W. Förstner, H. Ruwiedel (eds.) Robust Computer Vision: Quality of Vision Algorithms, pp. 59-78. Wichmann, Karlsruhe (1992)

4. Basseville, M.: Distance measures for signal processing and pattern recognition. Signal Process. 18(4), 349-369 (1989). DOI http://dx.doi.org/10.1016/0165-1684(89)90079-0

5. Baudrier, E., Millon, G., Nicolier, F., Ruan, S.: Binary-image comparison with local-dissimilarity quantification. Pattern Recognition 41(5), 1461-1478 (2008)

6. Belaroussi, B., Benoit-Cattin, H., Odet, C.: Scalable discrepancy measures for segmentation evaluation. In: ICIP (1), pp. 785-788 (2002)

7. Beucher, S., Lantuejoul, C.: Use of watersheds in contour detection. In: International Workshop on Image Processing: Real-time Edge and Motion Detection/Estimation, Rennes, France. (1979)

8. Brouwer, L.E.J.: Beweis des jordanschen satzes für n-dimensionen. Math. Annalen 71, 314-319 (1911)

9. Capasso, V., Micheletti, A.: Stochastic geometry and related statistical problems in biomedicine. In: Complex Systems in Biomedicine, pp. 35-69. Springer Milan Ed. (2006). DOI 10.1007/88-470-0396-2 2

10. Chabrier, S., Laurent, H., Rosenberger, C., Emile, B.: Comparative study of contour detection evaluation criteria based on dissimilarity measures. J. Image Video Process. 2008(2), 1-13 (2008). DOI http://dx.doi.org/10.1155/ $2008 / 693053$

11. Cárdenes, R., de Luis-García, R., Bach-Cuadra, M.: A multidimensional segmentation evaluation for medical image data. Computer Methods and Programs in Biomedicine 96(2), 108 - 124 (2009). DOI DOI:10.1016/j.cmpb.2009.04. 009

12. Debayle, J., Gavet, Y., Pinoli, J.C.: General Adaptive Neighborhood Image Restoration, Enhancement and Segmentation, LNCS: Image Analysis and Recognition, vol. 4141, chap. Image Restoration and Enhancement, pp. 29-40. Springer Verlag (2006). DOI 10.1007/11867586_3

13. Deza, M.M., Deza, E.: Dictionary of distances. Elsevier (2006)

14. Dirichlet, G.: Über die reduktion der positiven quadratischen formen mit drei unbestimmten ganzen zahlen. J. Reine Angew. Math. 40, 209-227 (1850)

15. Federer, H.: Geometric measure theory. Springer-Verlag (1969)

16. Fu, J.H.G.: Tubular neighborhoods in Euclidean spaces. Duke Math. J. 52, 1025-1046 (1985). DOI 10.1215/ S0012-7094-85-05254-8

17. Gavet, Y., Pinoli, J.C.: Visual perception based automatic recognition of cell mosaics in human corneal endothelium microscopy images. Image Anal. Stereol. 27, 53-61 (2008)

18. Hausdorff, F.: Grundzuege der Mengenlehre. Viet, Leipzig (1914)

19. Jaccard, P.: Étude comparative de la distribution florale dans une portion des alpes et des jura. Bulletin de la Société Vaudoise des Sciences Naturelles 37, 547-579 (1901)

20. Kerautret, B., Lachaud, J.O.: Multi-scale analysis of discrete contours for unsupervised noise detection. In: IWCIA, pp. 187-200 (2009)

21. Klette, R., Rosenfeld, A.: Digital geometry. Morgan Kaufmann (2004)

22. Lebesgue, H.L.: Sur la mesure des grandeurs. L'enseignement mathématique (1935)

23. Marczewski, F., Steinhaus, H.: On a certain distance of sets and the corresponding distance of functions. In: CoIloquim Mathematicum, vol. 6, pp. 319-327 (1958)

24. Marr, D., Hildreth, E.: Theory of edge detection. Proceedings of the Royal Society of London. Series B, Biological Sciences (1934-1990) 207(1167), 187-217 (1980)

25. Martin, D.R.: An empirical approach to grouping and segmentation. Ph.D. thesis, EECS Department, University of California, Berkeley (2003)

26. Michel, O., Baraniuk, R., Flandrin, P.: Time-frequency based distance and divergence measures. In: Time-Frequency and Time-Scale Analysis, 1994., Proceedings of the IEEE-SP International Symposium on, pp. 64-67 (1994). DOI 10.1109/TFSA.1994.467363

27. Micheletti, A., Capasso, V.: The stochastic geometry of polymer crystallization processes. Stochastic Analysis and Applications 15(3), 355-373 (1997). DOI 10.1080/07362999708809481

28. Minkowski, H.: Volumen und Oberfläche. Mathematische Annalen 57, 447-495 (1903)

29. Morgan, F.: Geometric measure theory. Academic Press, San Diego, CA, USA (1995)

30. Nguyen, T.P., Debled-Rennesson, I.: Curvature estimation in noisy curves. In: CAIP, pp. 474-481 (2007)

31. Nikodým, O.M.: Sur une généralisation des intégrales de M. J. Radon. Fund. Math. 15, 131-179 (1930)

32. Okabe, A., Boots, B., Sugihara, K.: Spatial tessellations: concepts and applications of Voronoi diagrams. John Wiley \& Sons, Inc., New York, NY, USA (1992)

33. Philipp-Foliguet, S., Guigues, L.: Évaluation de la segmentation d'images: état de l'art, nouveaux indices et comparaison. Traitement du signal 23(2), 109-124 (2006)

34. Pompeiu, D.: Sur la continuité des fonctions de variables complexes (thèse). Annales de la faculté des sciences de Toulouse Sér. 2 7, 265-315 (1905)

35. Román-Roldán, R., Gómez-Lopera, J.F., Atae-Allah, C., Martínez-Aroza, J., Luque-Escamilla, P.L.: A measure of quality for evaluating methods of segmentation and edge detection. Pattern Recognition, 34(5), 969-980 (2001)

36. Rosin, J., West, G.: Segmentation of edges into lines and arcs. Image and Vision Computing 7(2), 109-114 (1989)

37. Santini, S., Jain, R.: Similarity is a geometer. Multimedia Tools Appl. 5(3), 277-306 (1997)

38. Stoyan, D., Kendall, W.S., Mecke, J.: Stochastic geometry and its applications. Wiley (1995) 
39. Strasters, K.C., Gerbrands, J.J.: Three-dimensional image segmentation using a split, merge and group approach. Pattern Recognition Letters 12(5), 307-325 (1991)

40. Tversky, A.: Features of similarity. Psychological Review 84(4), 327-352 (1977)

41. Tversky, A., Gati, I.: Similarity, separability and the triangle inequality. Psychological Review 89, 123-154 (1982)

42. Veltkamp, R.: Shape matching: similarity measures and algorithms. In: Shape Modeling and Applications, SMI 2001 International Conference on., pp. 188-197 (2001)

43. Veltkamp, R.C., Hagedoorn, M.: Shape similarity measures, properties and constructions. In: VISUAL '00: Proceedings of the 4th International Conference on Advances in Visual Information Systems, LNCS, vol. 1929, pp. 467-476. Springer-Verlag, London, UK (2000)

44. Villa, E.: On the outer minkowski content of sets. Annali di Matematica Pura ed Applicata 188, 619-630 (2008). DOI $10.1007 / \mathrm{s} 10231-008-0093-2$

45. Voronoi, G.: Nouvelles applications des parametres continus a la theorie des formes quadratiques. premier mémoire: sur quelques propriétés des formes quadratiques positives parfaites. Journal für die Reine und Angewandte Mathematik 133, 97-178 (1907)

46. Voronoi, G.: Nouvelles applications des parametres continus a la theorie des formes quadratiques. deuxième mémoire: Recherches sur les parallélloèdres primitives. Journal für die Reine und Angewandte Mathematik 134, 198-287 (1908)

47. Voronoi, G.: Nouvelles applications des parametres continus a la theorie des formes quadratiques. deuxième mémoire: Recherches sur les parallélloèdres primitifs, seconde partie: Domaines de formes quadratiques correspondant aux différents types de parallélloèdres primitives. Journal für die Reine und Angewandte Mathematik 136, 67-181 (1909)

48. Yasnoff, W.A., Mui, J.K., Bacus, J.W.: Error measures for scene segmentation. Pattern Recognition 9(4), 217 - 231 (1977). DOI DOI:10.1016/0031-3203(77)90006-1

49. Zhang, H., Fritts, J.E., Goldman, S.A.: Image segmentation evaluation: A survey of unsupervised methods. Computer Vision and Image Understanding 110(2), 260-280 (2008)

50. Zhang, Y.J.: A survey on evaluation methods for image segmentation. Pattern Recognition 29(8), 1335-1346 (1996) 